\title{
Ultra-precise quantal timing: Evidence from simultaneity thresholds in long-range apparent movement
}

\author{
HANS-GEORG GEISSLER, FRANK-UWE SCHEBERA, and RAUL KOMPASS \\ University of Leipzig, Leipzig, Germany
}

\begin{abstract}
Conditions for the disappearance of long-range apparent movement were investigated. In an experiment on beta motion, critical interstimulus intervals (ISIs) of downward simultaneity thresholds for stimuli presented in continuous alternation were determined for exposure durations (EDs) varying from 3 to $160 \mathrm{msec}$. Each subject performed each test twice. Data were collected in three sessions, each for one of three angular separations $\left(3^{\circ}, 6^{\circ}\right.$, and $\left.12^{\circ}\right)$ and the full set of EDs. The distribution of critical ISIs collapsed across subjects, EDs, and angular separations shows sharp maxima at regular distances within a range of $0-110 \mathrm{msec}$ ISI. Significant or near-significant peaks were found at ISIs of 5, 9, 22, 27, 43,55 , and $107 \mathrm{msec}$. Although mean critical ISIs shifted with spatial separation, no essential shift of the main maxima occurred. Evidence of a periodic modulation with a period duration of $4.5 \mathrm{msec}$ was obtained from the distributions of differences between critical ISIs of the first tests and their replications, which exhibit extremely low standard deviations $(<10 \mathrm{msec})$. These results agree well with previous analyses (Geissler, 1987, 1992) that led to a taxonomic model of quantal timing, briefly summarized in this paper. Further consequences are discussed and related to earlier developments (Geissler, 1991, 1992, 1997).
\end{abstract}

In this paper, we consider simultaneity thresholds of apparent movement as a tool for exploring phenomena of temporal quantization in perception on the millisecond level of real time. Before introducing the experimental setup, we present a brief outline of the theoretical rationale from which our expectations about fine-grained temporal structures in perception derive.

\section{Motivation}

Whether or not mental processes exhibit a discrete quantal temporal fine-structure is an old question in psychology. But to date, none of the proposed answers has proved to be satisfactory. Early theorizing on the issue was based on the notion of a psychological moment as a universal building block of mental time (Stroud, 1956; Baer, 1864). Evidence from the last four decades, however, indicates that approaches adopting universal principles for mental timing cannot safely assume one unique basic unit of time, if a theory of this type can be maintained at all (Block, 1990; McReynolds, 1953; for an over-

This work was supported by Grant Gei $678 / 5-2$ of the German research organization DFG. We thank Christian Kaernbach, Stephen W. Link, Axel Mecklinger, Michel Treisman, and an anonymous reviewer for criticism and useful proposals. We are particularly grateful to Stephen W. Link for help in the final wording of the paper. The authors wish to express their deep gratitude to the late Eckard Scheerer for gracious support. Correspondence concerning this article should be addressed to H.-G. Geissler, Institut für Allgemeine Psychologie, Universität Leipzig, Seeburgstr. 14-20, 04103 Leipzig, Germany (e-mail: geissler@rz.uni-leipzig.de). view, see Neumann, 1983). There are two major reasons to doubt a simple time atom assumption. First, this assumption is in difficulty when attempting to explain the emergence of a large variety of empirically established critical periods of different durations. Second, it is incapable of giving a plausible account of obvious differences in specific qualitative characteristics of these periods. This deficiency seems even more damaging to the classical concept, because it cannot easily be removed by additions to it that would permit for more than one time unit. The problem is that, in the variety of observed critical periods, quantitative and qualitative properties do not seem to combine very well. Thus, some of the possible candidates for critical periods are of the type of lower absolute thresholds but, at the same time, differ strongly in their values. Examples are the thresholds for the perception of the successiveness of two brief sound signals and for that of their temporal order (see, e.g., Hirsch, 1975; Hirsch \& Sherrick, 1961). Other suggested candidates are upper thresholds, in the sense of temporal boundaries that, toward longer durations, separate regions of one type of phenomena from other, qualitatively distinct ones. A wellknown example is the upper boundary of the time of presence (see, e.g., Fraisse, 1966), the critical time separating experienced from remembered events. But psychophysical laws for the judgment of duration in this case extend across the assumed boundary, showing breaks at quite other locations (Eisler, 1990). Yet other proposed critical periods share features with conventionally measured difference thresholds. Examples are critical periods in duration discrimination that strongly argue for quantal properties in mental timing (see, e.g., Kristofferson, 1980, 1990). How- 
ever, the critical values in this case do not directly derive from a judgmental criterion. Rather, they are given by intervals of constant discrimination between breaks arising in the original Weber function after prolonged practice, breaks that, in defining the lower and upper limits of the corresponding intervals, by themselves demarcate other quantal values.

Whereas in these examples of specifying characteristics, the measurement of critical periods depends on subjective criteria of experienced time and even on statistical definitions, there have been several attempts to include findings that are based on more objective measures, such as the locations of discontinuities in absolute thresholds of vibration (Békésy, 1936), distinct modes in reaction time distributions (e.g., Dehaene, 1993; Jokeit, 1990; Latour, 1967; Pöppel, 1970), or specific shift patterns in duration judgments and in the spectra of electroencephalograph oscillations as a function of frequency of visual driving (see Treisman et al., 1994; also, for further references). In these latter cases, there is no obvious criterion available for attributing the measured periods to one of the above categories. Part of the collected evidence points to the existence of entire series of lawfully related critical periods, giving rise to proposals of superordinate laws of temporal quantization (e.g., Békésy, 1936; Kristofferson, 1980; Latour, 1967). Although these series of periods span ranges that exhibit, at least, definitive lower boundaries, there seem, with the exception of Kristofferson's (1980) data, to exist no direct correspondences to thresholds of perceived time as measured by classical psychophysical methods.

In this paper, we consider the concept of finite adaptive ranges of critical periods as a possible solution to the difficulties and conflicting views arising in connection with quantal timing. This notion was introduced as a key concept to portray achievements of quantal timing (Geissler, 1987, 1990; see, also, Geissler, 1985b). It is based on the general view that quantal timing represents a tool for the brain to ensure fast, temporally extremely precise communication among neural units that, depending on their function and phylogenetic origin, may differ widely in their temporal characteristics-a tool that, at the same time, provides the means for highly adaptive control in processing the streams of information within and between these units. Ranges are thought to reflect invariant dynamic properties of this timing system as a whole, rather than characteristics of a particular processing unit or of a special timing mechanism, like a central clock in the brain.

To comprehend the general idea, consider the ordered set of arbitrarily fixed integer numbers, $M 1=(1,2,3,4$, $5,6,7,8)$. The possible ratios among these numbers exemplify the quantal relations among a corresponding collection of real critical periods. In particular, the 1:8 ratio between the smallest and the largest numbers of $M 1$ maps the extension of a given range. Range adaptation in this instantiation implies multiplication of all the members of $M 1$ with a certain stretching factor $q$. To ensure compatibility of timing among subunits of the processing system, $q$ is assumed to be an integer too, at least as long as these units are coupled. In this way, we get larger ranges-for example, $M 2=(2,4,6,8,10,12,14,16)$ and $M 3=(3,6,9,12,15,18,21,24)$. Note that, according to this construction, sets of ranges conform with Weber's law as the possible distances among numbers increase proportional to the range extensions. In comparing ranges, the above issue of lacking or wrong correspondences between duration quantity and qualitative aspects of content attributable to periods does not any longer pose a problem: Some subunits (think of the auditory and visual receptor systems) may differ strongly in their temporal resolution and, therefore, lock into the timing system with ranges of quite different extensions. Two particular tasks - for example, unordered and ordered discrimination - may, for whatever reasons, require adjustment of different lower bounds and, thus, adapt to ranges of different range extensions. Also, periods of different denotation may well coincide in duration and still carry different denotations. (The numbers 2, 4 , and 6, for example, are in common to $M 1$ and $M 2$, but they still can belong to different ranges.)

A possible objection to the concept, as outlined so far, is not that it is too restrictive but, rather, that it may allow too much. But this would seem true only for the above rough outline. Building on the range rationale, in the following we refer to a more elaborated framework, the taxonomic time quantum model (TQM; see Geissler, 1987, 1990, 1992; also, Geissler, 1985b), which provides much richer constraint.

As will be seen, the basis for this is twofold: First, the range concept as a structure to map universal adaptive timing in the brain makes sense only if the temporal properties of this system reduce to a few numerical invariants. According to TQM, one of these invariants is the relative extension of the ranges, as reflected by the ratio between the largest and the smallest possible periods. In terms of classical concepts, this is equivalent to saying that, in a given state of range adjustment, there is a fixed maximal number of just noticeable differences in temporal discrimination. Another numerical invariant derives from the definitive lower limit for the timing of the interneuronal cross talk. Specifically, it is assumed that, in the global timing of neural mass activity, this constraint unifies to a precise absolute lower limit for the smallest possible time period within a range. Both invariants together--that of the limitation of relative range extension and that of an absolute lower bound-constrain the possible quantal time structures very strictly.

Second, these invariants are no ends in themselves. They rather reflect dynamic properties of neural organization that interact in the determination of concrete occurrences of quantal time periods. TQM, in its current form, includes two provisional semi-empirical specifications of this interaction. Most fundamentally, it introduces the idea that the upper limitation of ranges follows as a necessary consequence from the property that the corresponding processes are entrained and are rising and expiring according to the same general laws, on any level of timing. From this property, the TQM predicts increas- 
ing fuzziness in the periods of a quantal activity during epochs of longer durations. A second specification is a hypothesis about spontaneous preferences that occur when situational factors do not restrict the admissible options. It says that, under these circumstances, the transition occurs from a primary state of chaotic activity into a state of quantal timing with equal probabilities to each of the admissible states of quantal timing, including those that result from the organization of co-occurring periods. This specification again provides means for new, testable predictions.

In the following, to give a sufficiently detailed introduction to these TQM claims, we refer to a reformulation, in terms of oscillator processes, that translates, at least partially, into physiological interpretations (Geissler, 1991, 1992, 1997; see, also, Geissler, 1985a).

\section{Basic Time Quantum Assumptions}

In the oscillator representation of TQM, ranges of critical periods are conceived as nested sets of lawfully related oscillations (Geissler, 1990; see, also, Geissler, 1985a, 1991). For specification of the main postulates, consider the following hypotheses.

1. Coherence length. Figure 1 illustrates a first type of assumed lawful relations by the behavior of a package of entrained coherent oscillations of slightly differing frequencies that desynchronize after $M$ periods in sequence (in the example, arbitrarily put to 12 ), so that the original period can no longer be identified. $M$ is referred to as coherence length. For oscillations of a period of duration $d$, a breakdown of timekeeping on this level of resolution follows after a period of $D=M \cdot d$, the coherence duration.

Note that, with the hypothesis of limited temporal coherence, the TQM agrees with basic empirical quantum phenomena. In particular, it agrees with quantal discrimination attained after prolonged training (see, e.g., Kristofferson, 1980; see, also, Kristofferson, 1990, for an overview), which as a function of base duration, yields intervals of approximately constant discriminability, followed by sudden rises. The latter can be interpreted as indicating that the process of discrimination switches to slower oscillations desynchronizing at later points of time. For a recent application of a closely related concept in other areas, the reader is referred to the microgenetic study of van Leeuwen and Bakker (1995). On a more theoretical level, the postulate offers a general principle for relating different kinds of critical periods. To see this, we have to realize that, for a given oscillation of a quantal time period $T Q$, there exists just one slowest oscillation of period duration, $T M=M \cdot T Q$, to which it can connect, because this oscillation traverses, within the coherence interval of the fast oscillation, the necessary minimum of but one full period. $T Q$ and $T M$ may, therefore, be considered as lower and upper bounds of ranges of period durations $t$, or simply ranges, spanned by them in such a way that $T Q \leq t \leq T M=M \cdot T Q$.

To model real brain processes for the coherence length, the value $M=30$ was adopted as an ideal upper bound, which in actual performance, however, need not always be reached. A bound $N \leq M$ becoming operative in a particular instance will be designated as operative bound. Evidence supporting the proposed value of $M$ has been summarized in Geissler (1997).

2. Hierarchy formation. To accommodate further lawful relations observed among periods, the hypothesis was advanced that periods within a given range tend to form hierarchies of preferred periods, characterized by integer ratios of admissible period durations $T$ (Geissler, 1985a). In the oscillator interpretation, this is equivalent to the assumption that cooperating oscillations tend to establish harmonic frequency relations and maximum phase coincidences (phase locking or tuning) among one another (Geissler, 1991, 1997; see Lisman \& Idiart, 1995; Treisman et al., 1994; Weiss, 1992, for independent developments). Figure 2 illustrates how hierarchy formation applies to a range of periods in the ideal case of a complete within-range hierarchy. In the example, the longest period at the top, which is 24 times that of the shortest at the bottom, becomes sequentially segmented by subordinate oscillations into periods of $1 / 2,1 / 4$, and $1 / 8$ of its duration and finally splits into 24 of the shortest periods.

As can be seen from the example, it is a consequence of hierarchy formation that, as soon as it occurs, certain period durations within a given range become preferred. Furthermore, as we will see below, preferred periods that are predicted at regular places within a range may differ in their degree of preference, or prominence, as they may occur as part of different hierarchies related to one and

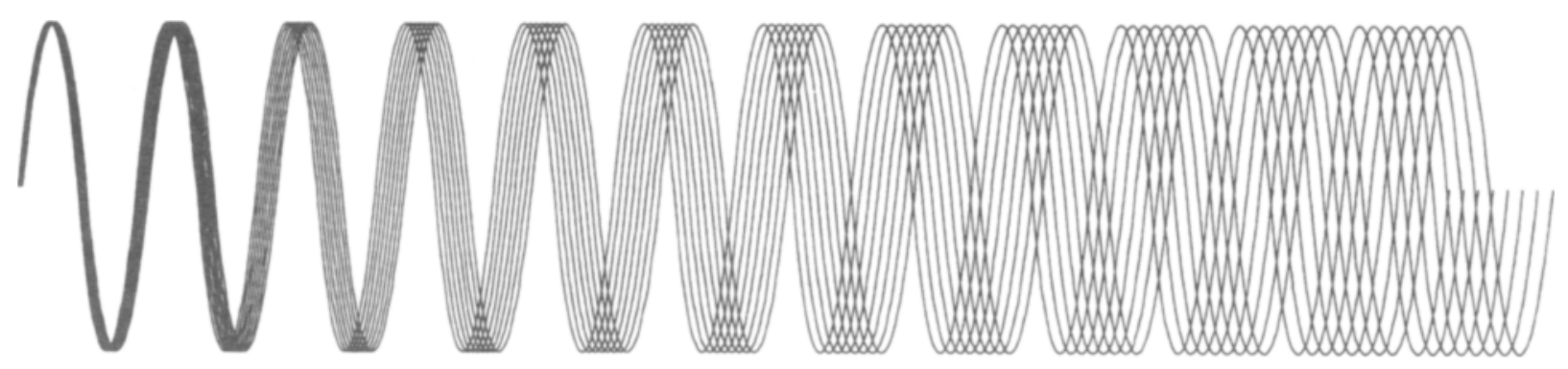

Figure 1. Illustration of coherence constraints: Oscillations of slightly differing period durations, synchronized at the start, get out of phase. In the case of random differences, the coherent activity is lost forever. The example demonstrates a near washout of periodicity within the arbitrarily fixed number of 12 oscillations. 


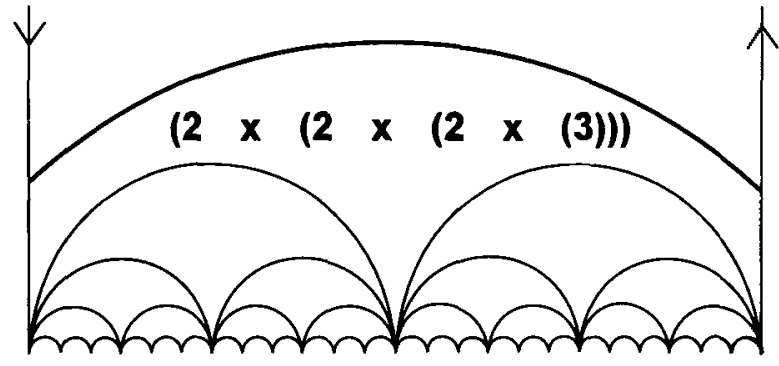

Figure 2. Illustration of a complete within-range synchronization hierarchy. Each of the periods between the shortest and the longest is an integer multiple of a next shorter and an integer fraction of a next larger period. The inscribed multiplication expression gives an easy description of this structure.

the same base interval or of hierarchies related to different intervals. Ideally, in the following, we will assume that hierarchies include only periods that are integer multiples of the shortest period of a range. These are referred to as dense hierarchies. It should, however, be noted that there is evidence to suggest that, in reality, constraints may often be weaker. It appears, for example, that the fastest oscillations may show period durations, $t \geq T Q$, that are integer fractions of some longest reference period $M \cdot T Q$ but that fail to be small multiples of $T Q$.

3. Base-level range and range hierarchies. Up to this point, the hypotheses considered represent structural constraints that imply statements about relations. In this sense, even the numerical invariant (theoretical parameter) $M$ implies a relative statement on the ratio between the durations of the largest to the shortest period within a nested set of periods. This situation is changed by the introduction of the TQM hypotheses (1) that there exists a shortest possible or base-level range, $R_{1}$, and (2) that other ranges, $R_{q}$, are related to it by integer stretching $R_{q}=$ $q \times R_{1}$, for which a numerical estimate of $T Q$ can be provided. Hypothesis 1 implies the existence of a shortest period $T Q_{0}$, defined as an absolute lower bound or time quantum of critical durations involved in cognition. Checking hypothesis 2 requires firmly establishing the structure of $R_{1}$ and exploring the relationships to the second hypothesized range, $R_{2}$.

From various sets of data for $T Q_{0}$, a value of about $4.5 \mathrm{msec}$ was derived (see Geissler, 1987, 1992). Elementary periods of general significance of about this duration have also been proposed on the basis of physiological considerations (see Freeman, 1964, 1972, 1974; Hendrickson, 1972). In the following, we will adopt $4.57 \mathrm{msec}$ as an ideal estimate that is based on two independent calculations of the next larger unit, corresponding to the multiple $2 T Q_{0}$ (Bredenkamp, 1993; Geissler, $1985 b$ ). From this estimate and the coherence constraint as introduced above, it follows that $R_{1}$ expands over the interval $4.57 \mathrm{msec} \leq t \leq 137 \mathrm{msec}$. Consequently, $R_{2}$ (which is $2 \times R_{1}$ ) should expand over $9.13 \mathrm{msec} \leq t \leq$ $274 \mathrm{msec}$. The value of $T Q_{0}$ seems physiologically plausible, as it is of the same order of magnitude as cross-talk latencies (refractoriness plus interneuronal transmission time) of neurons that actively participate in cognitive processing within the cortex. To see this, one has to take into consideration manifestations of the components that are relevant with regard to the neural mass activity on which behavioral responses are based. The contribution of refractoriness is the absolute refractory period, which is about $1 \mathrm{msec}$. Synaptic transmission should add 0.5 to 1 msec (see Kandel, Schwartz, \& Jessell, 1995). The largest times to be accounted for come from interhemispheric transmission, independent of whether or not conduction along fibers is the only determining factor of these contributions. The most precise estimates of the behaviorally relevant measure of this component known to us are by Brysbaert (1994). They average to $2.4 \mathrm{msec}$. From these data, it can be concluded that no smaller fraction of $T Q_{0}$ that is consistent with those constituents of the TQM that are purely structural could, for physiological reasons, be accepted.

The analogy between the postulated time quantum $T Q_{0}$ and the classical psychological moment is obvious. Note, however, that $T Q_{0}$ is of much smaller magnitude than has ever been proposed in approaches based on the traditional moment hypothesis and, thus, does not have the operational meaning of a directly observable time threshold in perception.

With the hypotheses put forward in Sections 2 and 3 above, the TQM accounts for cascades of critical periods that are related by integer ratios, as was first observed by Békésy (1936) in vibration perception and Kristofferson (1980) in duration discrimination. By generalization, similar structures were predicted for reaction times in pattern recognition (Geissler, 1997). In the present paper, we apply the same rationale to elementary phenomena in vision, thereby expanding the application of the approach to sensory integration processes.

\section{Preferred Periods}

The main purpose of the present study is an experimental inquiry into fine structures of timing in a range of up to some $200 \mathrm{msec}$, a range that can be considered as characteristic of fast perceptual processing. In terms of TQM constructs, this implies that the ranges $R_{1}$ and $R_{2}$ should be involved. In the following, the straightforward assumption will be made that preferred empirical timing periods coincide with preferred periods predicted for $R_{1}$ or $R_{2}$. Because $R_{2}$ obtains from $R_{1}$ by a stretching factor of two, we will confine our considerations to $R_{1}$.

For the concrete calculation of theoretical degrees of preference, we have to take into account that periods of given durations, in terms of multiples $k$ of $T Q_{0}$, differ widely in their capacity to participate in hierarchy formation. For example, for $k=10$, there are six hierarchies containing a period of this duration, whereas for the prime number $k=19$, there is only one. Obviously, this property is the only one by which preference relations among admissible periods can be defined. For a start, we will therefore assume that, as long as situational constraints enforce no other preferences, combinatorial frequencies of occurrence within different hierarchies can be considered as suit- 


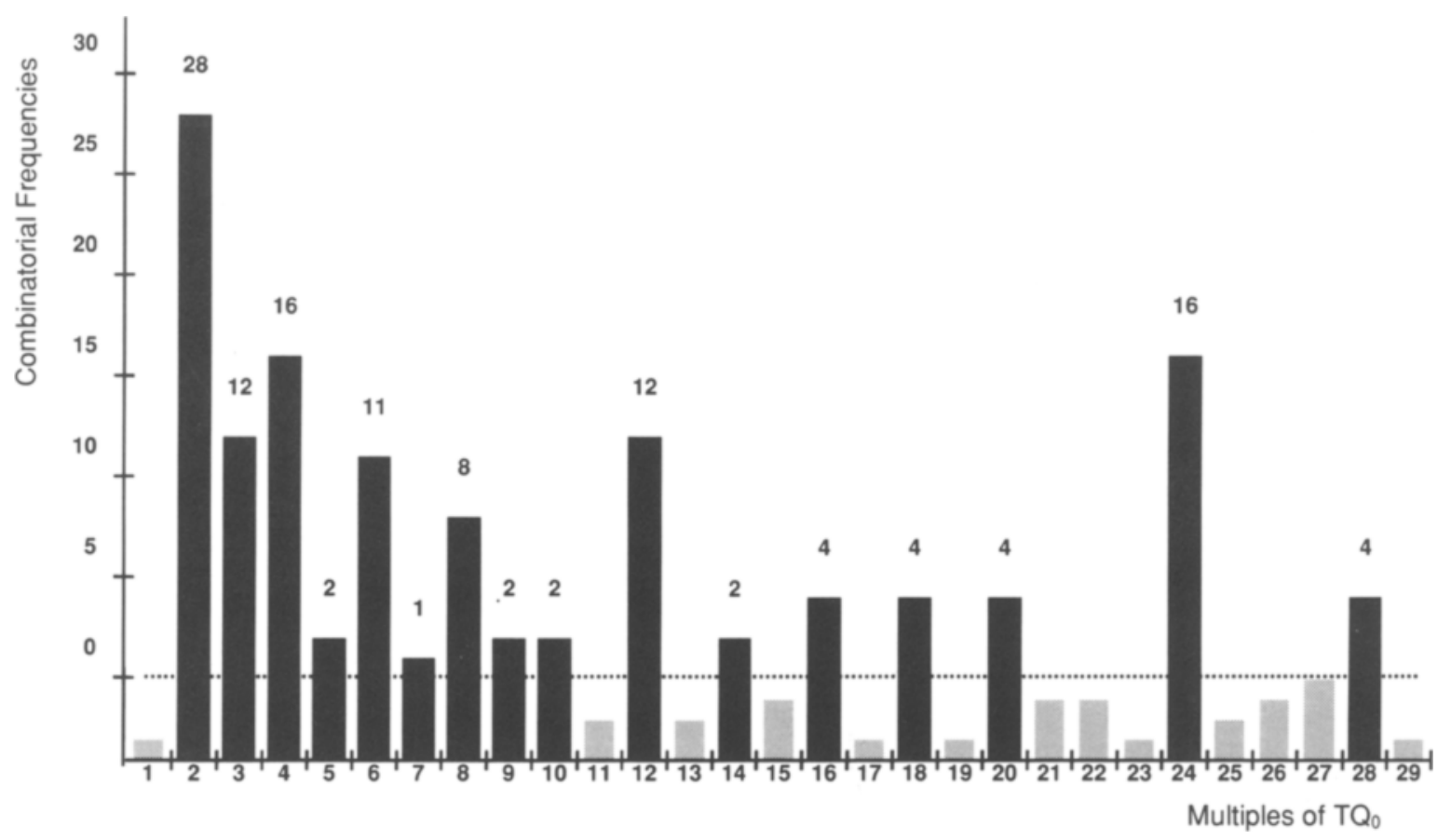

Figure 3. Period durations in multiples of $T Q_{0}$ and absolute combinatorial frequencies of their occurrence within different hierarchies. Further explanations appear in the text.

able measures of preference (or prominence) of any period under consideration. Figure 3 shows the a priori distribution deriving from this assumption if all multiples $k$ within a range are taken into consideration, with the exception of $k=30$, which was excluded for empirical reasons that will become obvious below. Putting a noise threshold of preference at four, we get the predictions in milliseconds: 9.1 (28), $13.7(12), 18.3(16), 22.8(2), 27.4(11), 36.6(8), 41.1$ (2), 45.7 (2), $54.8(8), 64$ (2), 73.1 (4), $82.3(4), 91.4(4)$, $109.7(16)$, and 128 (4), where the numbers in parentheses denote preference values above threshold.

\section{Choice and Specification of Paradigm}

Since for different reasons, none of the paradigms known to produce quantal effects could be expected to be suitable for demonstrating such fine-grained temporal patterns, apparent movement situations were chosen as prototypical paradigms. As compared with paradigms that use reaction time as a dependent variable, apparent movement has the advantage that relative timing of two or more stimuli is involved, rather than timing within stimulusresponse chains. The apparent movement paradigm may reduce or even eliminate variances caused by sensory and motor processing. A fundamental advantage, in comparison with duration discrimination, is that critical durations in apparent movement are marked by transitions between qualitatively distinct interpretations of stimulus displays, whereas in duration discrimination, subtle quantitative differences between the categories longer and shorter are separated by an interval of phenomenal equality. From this property, thresholds in apparent motion appeared to be particularly suitable for detecting discrete periods of timing on an extremely small scale of measurement. Another fairly important advantage is that, as will be seen below, the effects of two basic temporal parameters on the position of the threshold prove to be opposite. This allows, through manipulation of one of the parameters, the exploration of critical period durations for the other one in the full range of interest. We focused on a type of apparent movement today called beta motion, which was discovered by Exner (1875) and first systematically studied by Wertheimer (1912; see, also, Sarris, 1989). The phenomenon is perceived when two spatially separate spots of light are presented in succession. Under optimal timing, the impression is one of a single spot moving smoothly between the two sites of stimulus presentation. Wertheimer used what today is called a single-trial design - that is, just one pair of spots was presented in a trial. For these conditions, four phenomenologically differing stages and transitions between them have been described (see, also, Neuhaus, 1930). The first stage is one of simultaneity observed for smallest ISIs; the impression here is one of simultaneous appearance and disappearance of the two spots, each at its own location. The second stage is one of singular and dual movement for somewhat larger ISIs; subjective experience in this case comprises two different forms of seen partial movement-one in which the spot presented first phenomenally traverses half of the distance to the second spot, the latter at the same time remaining at its location (singular movement), and another in which the first spot traverses merely a smaller part of the entire distance, then disappears and reappears close to the phenomenally empty location of the second spot, then traversing the rest of the path. The third stage is one of $o p$ - 
timum, or beta, movement for interstimulus intervals (ISIs) $\sim 60 \mathrm{msec}$; under these conditions one spot appears to move smoothly as a clearly contoured object from one location to the other. The fourth stage is one of succession that is obtained with certainty for ISIs $>200 \mathrm{msec}$; here, the spots appear one after another at their two locations without any impression of movement between them.

Wertheimer (1912), reporting on influences on the occurrence of different stages and the transitions between them, listed exposure duration (ED), ISI, spatial separation, and stimulus intensity as primary factors and shape, color, relative orientation, and variables, such as set, fixation, and attention, as secondary factors. The first extensive parametric investigations were carried out by Neuhaus (1930), whose seminal work has until now remained one of the most extensive and detailed studies in the field. Like Wertheimer using a single-trial design, Neuhaus determined average stimulus onset asynchronies (SOAs) for transitions, both between apparent movement and perceived succession and between movement and perceived simultaneity as a function of ED and angular separation (AS). The results on these and other dimensions of variation studied during the subsequent history of research into the phenomenon (see Anstis, 1986; Neff, 1936) led us to focus on fundamental temporal determinants and the modulation of their impact by primary, nontemporal parameters. We decided to confine ourselves to $E D$, ISI (ISI = SOA - ED), and, as an easily controllable nontemporal variable, AS. Figure 4 schematically illustrates the dependency of critical ISI on ED for fixed AS. The upper curve in this picture represents the boundary between perceived movement and succession, the lower one that between movement and simultaneity. Because the area between these lines corresponds to the conditions in which perception of movement dominates, for each value of ED, these lines can be considered as representing the upper and lower thresholds of perceived movement (UT and LT in Figure 4), respectively.

Three additional aspects were taken into consideration when designing the experiment. First, we generally re- stricted ourselves to stimulus displays with angular separations $>2^{\circ}$, which most clearly belong to so-called long-range apparent movement (LAM). This category was introduced by Braddick $(1974,1980)$ and Anstis $(1978,1980)$ as one opposed to short-range apparent movement (SAM), to distinguish forms of seen movement that are very likely to differ in their underlying physiological mechanisms. Accumulated evidence suggests that LAM is a fully integrated fill-in process with a (yet unknown) high-level physiological correlate to which TQM postulates presumably apply. Support for this notion can be derived from the fact that, unlike SAM, LAM can be produced dichoptically (Shipley, Kenney, \& King, 1945) and that color contrast is sufficient for its appearance (Ramachandran \& Gregory, 1978). A later result particularly convincing of a high-level representation was obtained by Larsen, Farell, and Bundesen (1983), who, by varying stimulus-observer distances, showed for angles between $2^{\circ}$ and $5^{\circ}$ that it is not angular distance but reconstructed spatial distance that matters.

Second, to determine critical periods as precisely as possible, the stimulus presentation should guarantee maximum certainty of judgments about the transition and stability of transition points on replication. For this reason, conditions of continuous sequential alternation of stimuli were chosen. These turned out to yield no phenomena of incomplete motion, in the sense of stage 2 mentioned above, that could impair judgment and a more stable correspondence between phenomenal states and physical parameter combinations than can be obtained when using the classical mode of single-trial presentation.

Third, in addition, procedural details had to be fixed. The TQM approach clearly excludes procedures that (as, for example, does the method of constant stimuli in threshold determination) necessitate calculations complexly related to the raw data. We therefore decided to apply an adjustment procedure representing a version of the method of limits that permits sufficiently precise determination of the parameter values where the transition between the qualitatively distinct percepts occurs. To allow

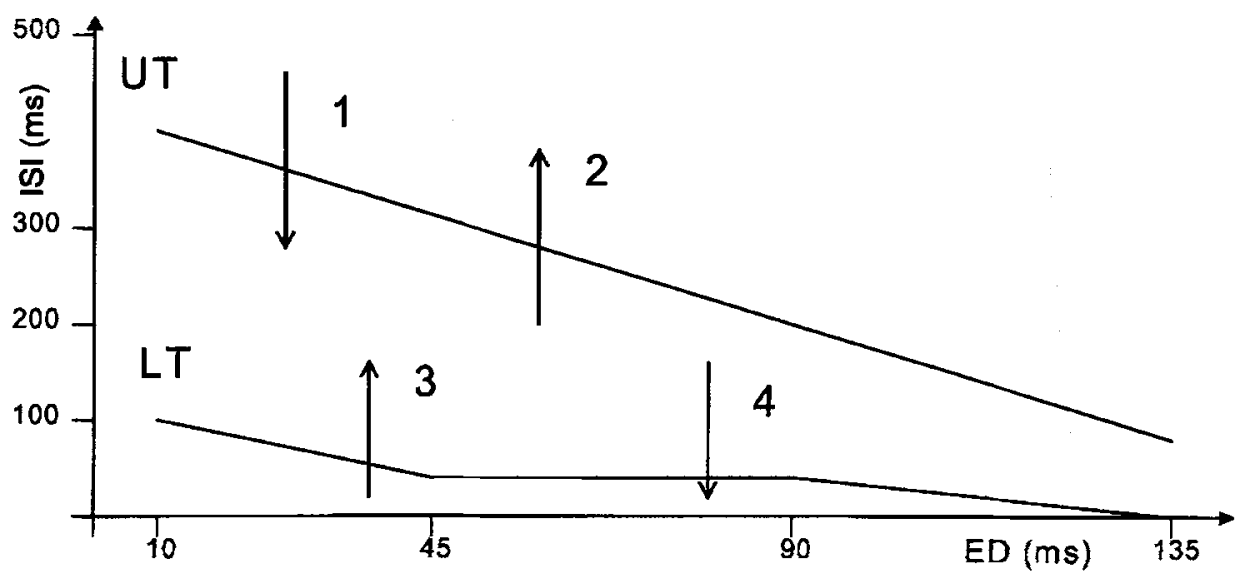

Figure 4. Schematic representation of regions of apparent motion, simultaneity, and successiveness in the exposure duration-interstimulus interval (ED-ISI) plane, with upper and lower thresholds (UT, LT) as boundaries. Arrows indicate the four cases of traversing these boundaries. 
for the detection of discrete fine structures in the data, the full distributions of single measurements had to be considered in the evaluation.

Seen movement is known to reappear after each breakdown. Because the point in time when this occurs varies under the impact of adaptation processes, in the reported experiment only the first transitions noted by the subjects were taken into consideration.

Pilot series were run in order to examine the preciseness of threshold determination for UT and LT through ascending and descending ISI variation. As a result, a rank order of intervals of first transition was established for each of the four cases corresponding to the numbers attached to the arrows in Figure 4. Thresholds for the transition from succession to beta motion (1) showed the highest variance, followed by the reverse transition (2) and the transition from simultaneity to motion (3). By far the smallest variances were found for transitions from seen motion to simultaneity (4), the downward simultaneity threshold. The latter condition was chosen because the experiment reported in the following was intended to serve as a first exploration of apparent movement from the point of view of ultra-fine-grain temporal properties.

\section{METHOD}

\section{Subjects}

Forty-six psychology students of ages 18-26, 31 female and 15 male, with normal or corrected-to-normal vision, served as subjects in the experiment in partial fulfillment of course requirements. With the exception of the 13 subjects indicated below, the subjects took part in the full experiment.

\section{Apparatus}

The stimuli were presented in a three-field exposure cabinet tachistoscope (Withers Ltd., VAT Reg. No. 2224234 10). The channels of the tachistoscope were designed for exposure of cards of 6 -in. horizontal and 4 -in. vertical extensions. Illumination could be varied within a range of 110-2,200 lux. In all the experimental series, illumination was constantly set at $70 \%$ of maximum intensity.

The controller of the tachistoscope was equipped with four timers, which permitted fixing EDs and ISIs with a maximum error of $\pm 2.5 \%$ of the chosen time value. Times necessary for the lamps to reach full intensity and full set-off were less than $0.2 \mathrm{msec}$. ISIs were varied by mechanical switching in steps of $1 \mathrm{msec}$.

\section{Material and Stimuli}

The subjects saw the rectangular stimulus cards at a distance of about 22-in. under maximum angles of $16^{\circ}$ and $10^{\circ}$ in the horizon- tal and vertical directions, respectively. Spots presented to induce beta motion consisted of white circular areas against a black background that subtended an angle of about $1^{\circ}$. The cards were illuminated only during epochs corresponding to the EDs.

\section{Design}

For 46 subjects, the design was a 2 (angular separation: $6^{\circ}$ and $\left.12^{\circ}\right) \times 12$ (EDs: $3,20,40, \ldots, 200,250 \mathrm{msec}$ ) complete factorial design with one replication for each condition. To ensure comparability with other studies, particularly those of Neuhaus, 33 of the subjects received one further angular separation $\left(3^{\circ}\right)$. The subjects participated in a sequence of sessions, during which angular separation was kept constant. EDs were randomly varied in each of the two sessions, with a minimum of trials before repetition of identical exposure durations.

\section{Procedure}

Each trial consisted of a period of display presentations with decreasing ISIs. After every trial, there was a short break for relaxing, and the next trial was verbally paced by the subjects. Before replication of ED conditions, there was a larger break of some $5 \mathrm{~min}$. Within a given trial, the spots (white circles) of the stimulus displays were presented in continuous sequential alternation, with one of the selected ED and ISI values iteratively produced from a start value. The start value was chosen well above threshold within a range of unquestioned perception of beta movement for each selected ED value. After setting the stimulus configuration at start value, the subjects were asked whether they saw a distinct movement. On affirmation, ISIs were smoothly diminished until the impression of a moving object suddenly disappeared. The subjects were instructed to respond verbally with "stop" as soon as this occurred. The corresponding ISI values were registered as critical ISIs. Adjusting ISIs at a sufficiently high rate proved to be necessary to avoid, as far as possible, confounding induced transition with spontaneous breakdown (Anstis, Giaschi, \& Cogan, 1985; Finlay \& von Grünau, 1987) - that is, spontaneous disappearance of seen motion well within established areas of stable apparent movement. To accomplish this, rates of change were chosen that were approximately proportional to the ISI value, ranging from rates of change of 5-10 $\mathrm{msec} / \mathrm{sec}$ for ISIs $>100 \mathrm{msec}$ to rates of $0.5-1 \mathrm{msec} / \mathrm{sec}$ for ISIs $<$ $10 \mathrm{msec}$. Systematic errors owing to latencies of the subjectexperimenter system were estimated to amount to maximally $2 \mathrm{msec}$ for critical ISIs $\approx 100 \mathrm{msec}$. They are negligible for smaller ISIs. The average duration of a trial, including a short break, was about $1 \mathrm{~min}$, the entire session lasting $30 \mathrm{~min}$ at the longest.

\section{RESULTS AND DISCUSSION}

The results of a three-way repeated measures analysis of variance, with critical ISIs as the dependent variable, based on the 33 subjects who ran through all conditions, are presented in Table 1. It revealed highly significant main

Table 1

Results of Analysis of Variance

\begin{tabular}{|c|c|c|c|c|c|c|c|c|}
\hline \multirow[b]{2}{*}{ Factors } & \multicolumn{2}{|r|}{ Effect } & \multicolumn{2}{|c|}{ Error } & \multirow[b]{2}{*}{$F$} & \multirow[b]{2}{*}{$p$} & \multicolumn{2}{|c|}{ Geisser-Greenhouse } \\
\hline & $d f$ & $M S$ & $d f$ & $M S$ & & & $\varepsilon$ & Corrected $p$ \\
\hline ED & 11 & $246,858.5$ & 352 & 147.7 & $1,671.3$ & .00000 & .739 & .00000 \\
\hline AS & 2 & $31,340.9$ & 64 & $1,006.6$ & 31.13 & .00000 & .481 & .00000 \\
\hline RP & 1 & 323.7 & 32 & 60.6 & 5.34 & .02746 & & \\
\hline $\mathrm{ED} \times \mathrm{AS}$ & 22 & 284.6 & 704 & 96.4 & 2.95 & .00001 & & \\
\hline $\mathrm{ED} \times \mathrm{RP}$ & 11 & 55.3 & 352 & 18.5 & 2.99 & .00082 & & \\
\hline $\mathrm{AS} \times \mathrm{RP}$ & 2 & 440.5 & 64 & 37.8 & 11.66 & .00005 & & \\
\hline $\mathrm{ED} \times \mathrm{AS} \times \mathrm{RP}$ & 22 & 30.5 & 704 & 16.5 & 1.85 & .01065 & & \\
\hline
\end{tabular}

Note-ED, exposure duration; AS, angular separation; RP, replication. 
effects of ED, AS, and replication (RP). All interactions between these factors were also found to be significant.

Mean critical ISIs for movement perception are plotted as functions of ED, with AS as parameter, in Figure 5. It shows a general decrease in critical ISI that is about half the corresponding increase in ISI. Critical ISIs are smaller for smaller AS. These systematic effects of AS are considerably smaller than those commonly obtained in single-trial designs. Critical SOAs (SOA $=E D+I S I)$ amount to about half of those of the classical Neuhaus study. In more detail, the observed average trend exhibits a plateau for EDs in the range from 3 to $40 \mathrm{msec}$ and some indication of a second leveling off in an interval from EDs of 100-140 msec. Pronounced plateaus in approximately this range, each followed by a drop, are evident in many of the corresponding functions for individuals but become washed out by averaging, owing to strong individual differences in their exact locations on the ED axis.

Figure 6 gives an impression of the existing individual differences that are particularly strong with regard to the dependencies of critical ISIs on AS. Whereas Figure 6A shows an example (Subject 23) where, for the three ASs, the functions obtained for the critical ISIs were practically identical in first and second testing, Figure 6B depicts an example (Subject 16) exhibiting nearly identical curves for critical ISI as a function of ED for first tests and replications that, however, strongly diverge for different ASs. An example (Subject 3) for generally much more irregular ED dependencies and low stability is shown in Figure 6C.

\section{Preferred Interstimulus Interval Modes}

To explore quantal properties of the distributions, frequencies of critical ISIs as a function of ED were cumulated across subjects. The results are depicted in the histograms of Figures $7 \mathrm{~A}-7 \mathrm{C}$ for $3^{\circ}, 6^{\circ}$, and $12^{\circ} \mathrm{ASs}$, respectively. These pictures show quite clearly that, to the shift of means across conditions of angular separation, there corresponds no essential shift of the modes of the distributions. Rather, as one should expect from the TQM assumptions, it is a "reshuffling" between constant modes of a multimodal distribution that accounts for the differences of the means. To some degree, the same property is visible also in the changes of the distributions with ED. Note that there is no mode at ISIs of around $137 \mathrm{msec}$, which, in the view of TQM, is compatible with the notion that the effective coherence length in all subjects was below the theoretical maximum of $M=30$. This necessitates and justifies exclusion of $k=30$ in calculating preference values,

To check on the specific predictions about preferred periods, we collapsed the distributions across ASs and EDs, because the location of preferred periods is expected to be independent of these parameters, despite their effect on the mean critical ISIs and despite any adaptation and learning that might occur during the experimental sessions. The result is depicted in Figure 8, which exhibits very clear properties of the expected type. As is commented on in the Appendix, from a comparison with simulated uniform distributions, highly significant peaks $(p<.005)$ in agreement with the predictions are found around critical ISI values of $9,22,27,43,55$, and $107 \mathrm{msec}$. Local maxima are also visible in the vicinity of the predicted places around $13.5,18,36,45,72$, and $90 \mathrm{msec}$, which, however, do not reach significance. Qualitatively, the distribution follows the expected concave trend (cf. Figure 3), with the highest frequencies being at the lower and upper bounds of the range of critical ISIs. There are also pronounced peaks at the $0-\mathrm{msec}$ ISI and around the 5-msec ISI $(.05<p<.06)$ that are not derivable from the above heuristic rationale, based on purely combinatorial considerations, but agree with the general assumptions on quantal timing given the temporal grid size within the range $R_{1}$. Namely, since with a certain probability, an induced breakdown of seen movement does not always occur at the most preferred ISIs, including $9 \mathrm{msec}\left(2 T Q_{0}\right)$, it is expected to occur at the

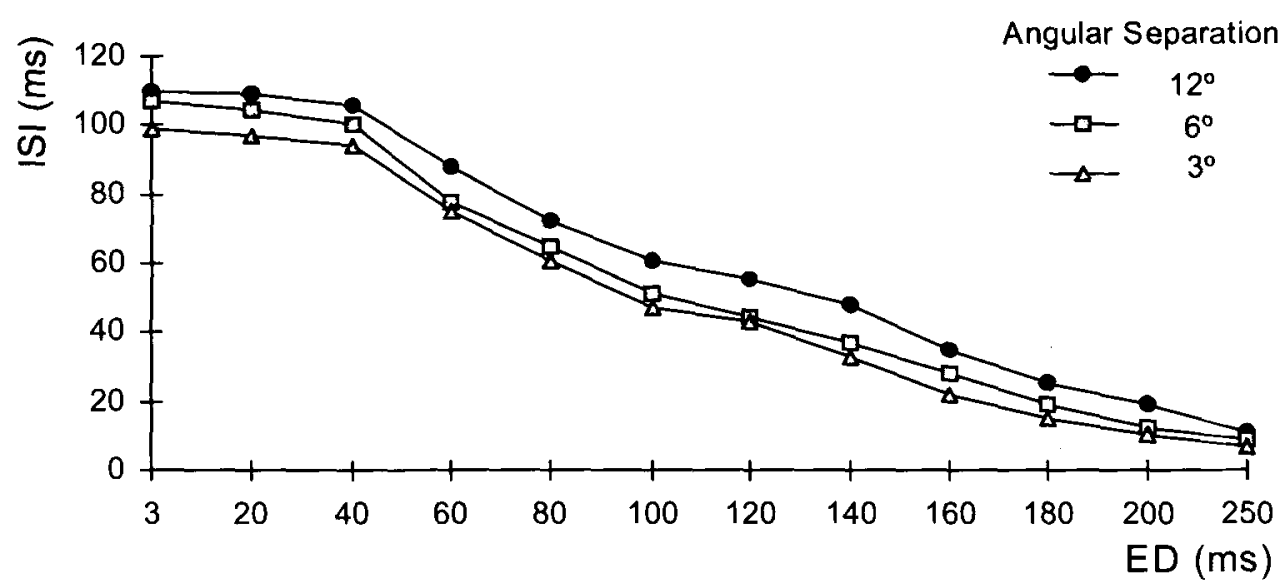

Figure 5. Mean critical interstimulus interval (ISI) for the simultaneity threshold as a function of exposure duration (ED) for 46 subjects. The parameter is angular separation $\left(3^{\circ}, 6^{\circ}, 12^{\circ}\right)$. 
A

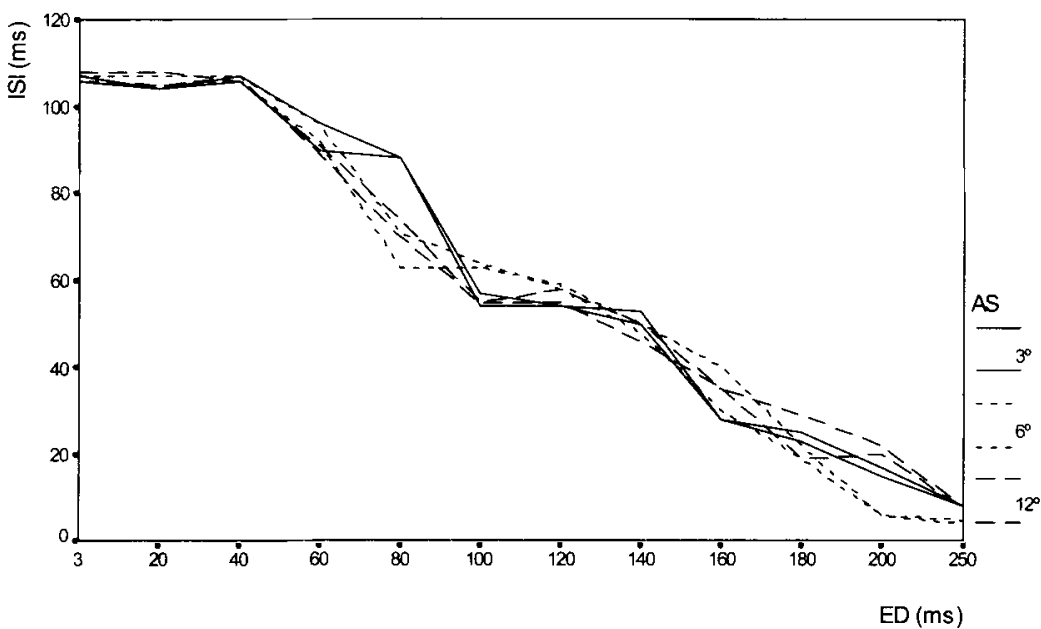

B

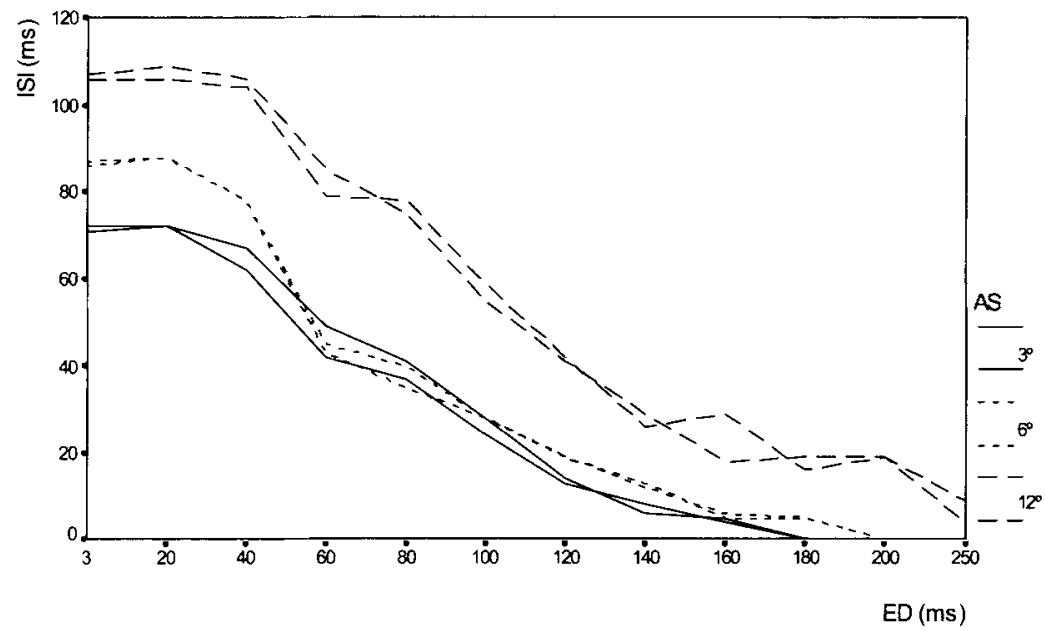

C

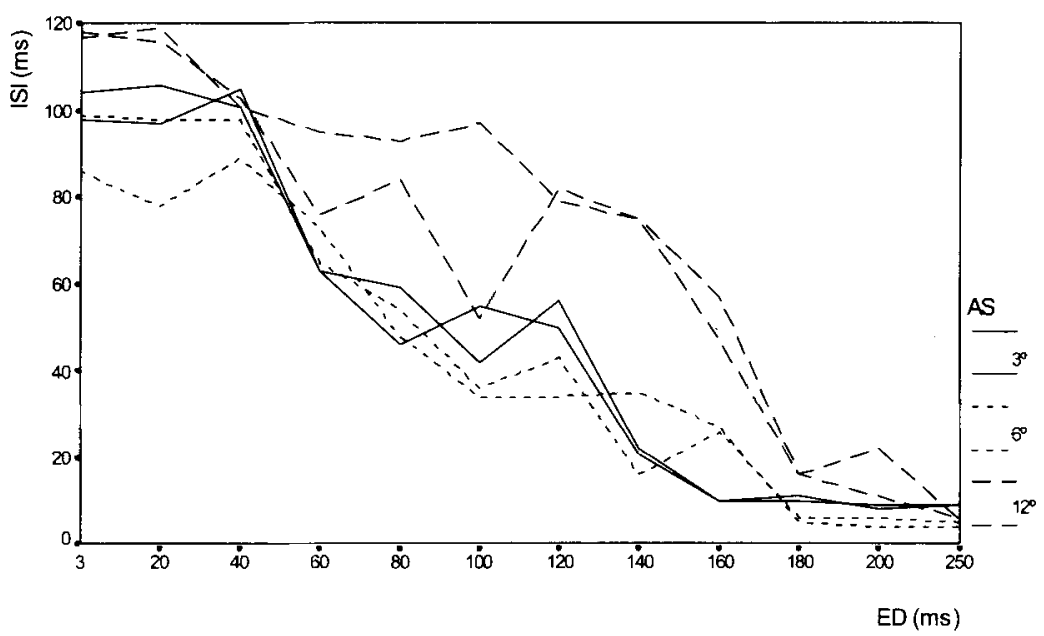

Figure 6. Examples of individual courses of critical interstimulus intervals (ISIs) as functions of exposure duration (ED), with angular separation (AS) as parameter. In the case of Figure $6 \mathrm{~A}$ (Subject 23 ), practically identical courses for all conditions were obtained. Figure 6B shows an example (Subject 16) of nearly exact replication of courses for identical ED conditions that strongly diverge for different angular separations. Data from a subject (Subject 3) exhibiting much more irregular courses of critical ISI plotted against ED and low stability are represented in Figure $6 \mathrm{C}$. 


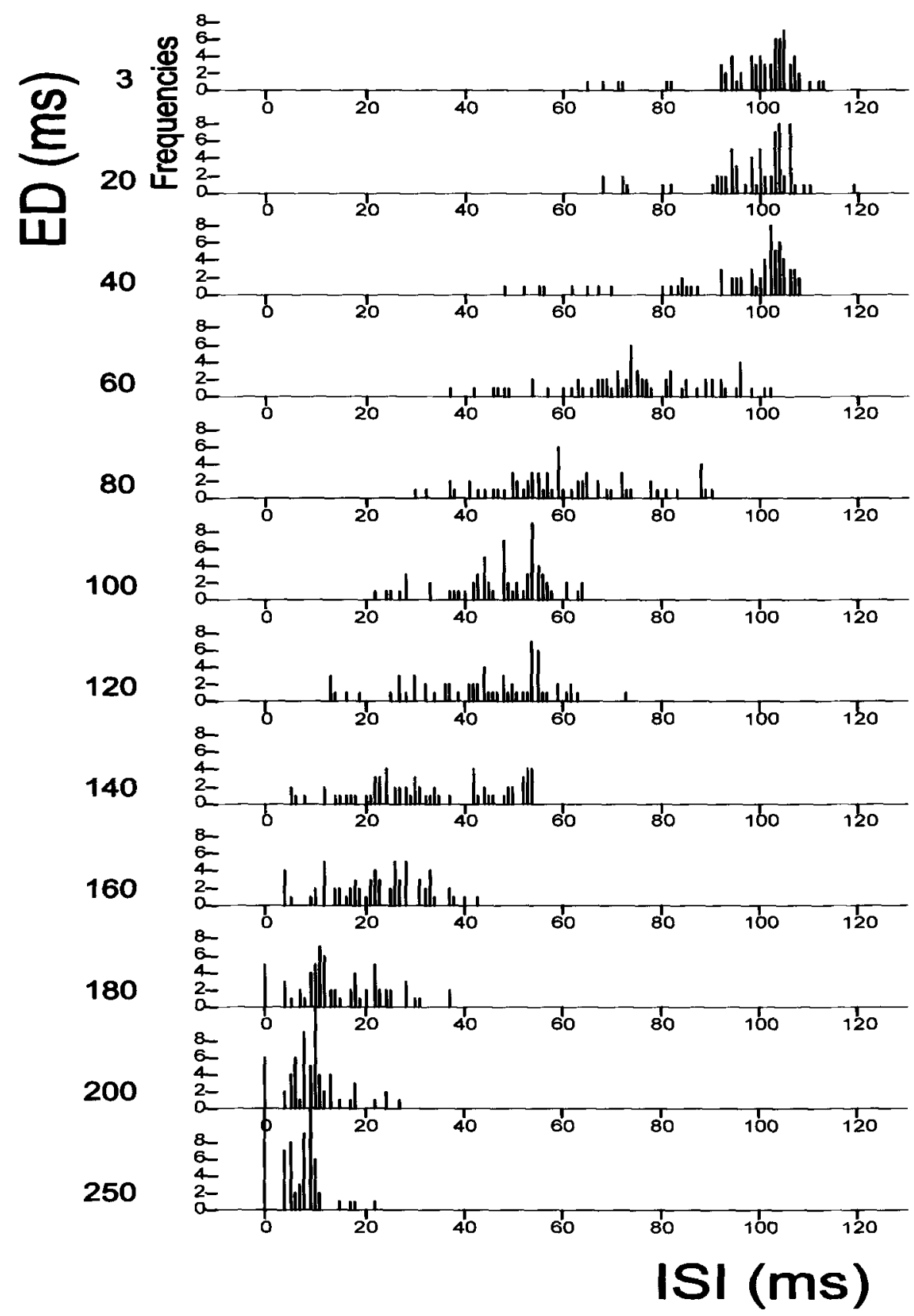

Figure 7A. Frequencies of critical interstimulus intervals (ISIs) for different exposure durations (EDs) and an angular separation of $3^{\circ}$, collapsed over subjects. Note that modes remain fixed across angular separations, even though means shift.

next lower quantal values, which, in the descending procedure, are ISIs of $4-5 \mathrm{msec}$ and, since negative ISIs were not included, of 0 msec.

One major conclusion from the described properties of the distributions concerns the status of ISI with respect to the temporal integration process underlying beta movement. If one adds to the ISI values the corresponding EDs, it becomes obvious that the strong regularities revealed are largely being destroyed. Indeed, as coinciding modes of critical ISIs get differentially displaced, depending on $E D$, no consistent rule holds for SOAs. At least for the continuous sequential alternation conditions investigated here, this clearly contradicts the widely accepted notion (see, e.g., Kolers, 1964) that SOA, considered as formation time, is the basic parameter underlying probability or strength of seen motion (see, also, Ganz, 1975).

\section{Preciseness of Timing and}

Local Quantum Properties

The above analysis is not completely informative about the precision of timing in a given trial. TQM predicts that it should be of the order of magnitude of $T Q_{0}$, the 


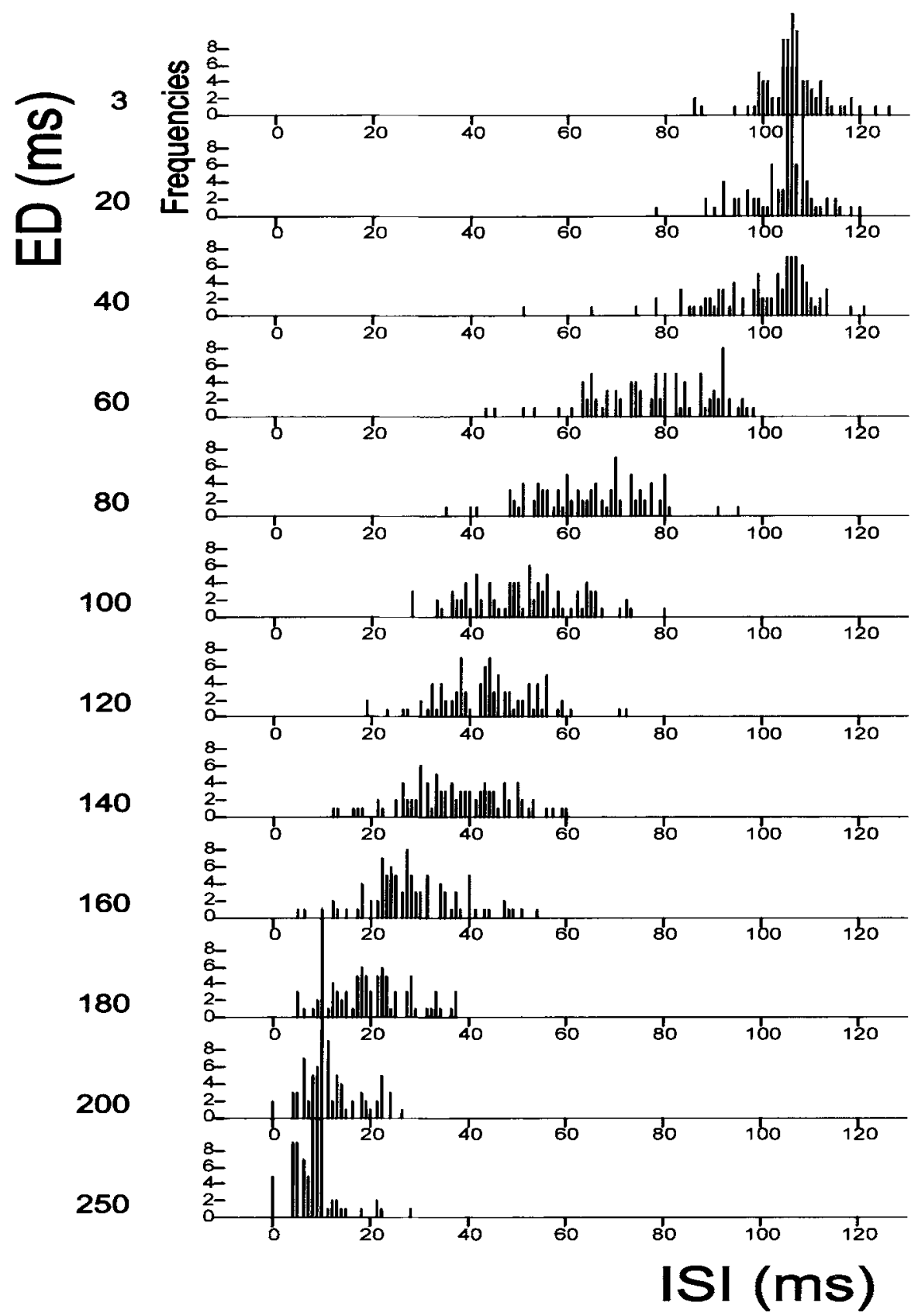

Figure 7B. Frequencies of critical interstimulus intervals (ISIs) for different exposure durations (EDs) and an angular separation of $6^{\circ}$, collapsed over subjects.

lower limit of resolution within $R_{1}$. To check on this assertion, the intraindividual distributions of differences of critical ISIs between the first and the second trials for otherwise identical conditions were calculated and collapsed over subjects and angular separations. Since no essential asymmetries were found in the distribution between negative and positive differences, signs were discarded from further analysis. Figure 9 shows the result. Note that the column for zero differences corresponds to only one interval of the original distribution, whereas the others are based on two of them.
The distribution shows side maxima at around ISI difference values, $\Delta \mathrm{ISI}$, of 9,13 , and $17 \mathrm{msec}$. For a statistical check of this property (see the Appendix), a two-step procedure was adopted. Initially, to obtain a good fit to an easily interpretable smooth curve, the individual distributions were collapsed across ASs and fit to an ideal distribution. A smooth overall approximation was obtained by averaging these idealized individual distributions. In another step, the overall function was modified by considering a normed average with a function comprising a periodic part. Then, $\chi^{2}$ was calculated, varying period 


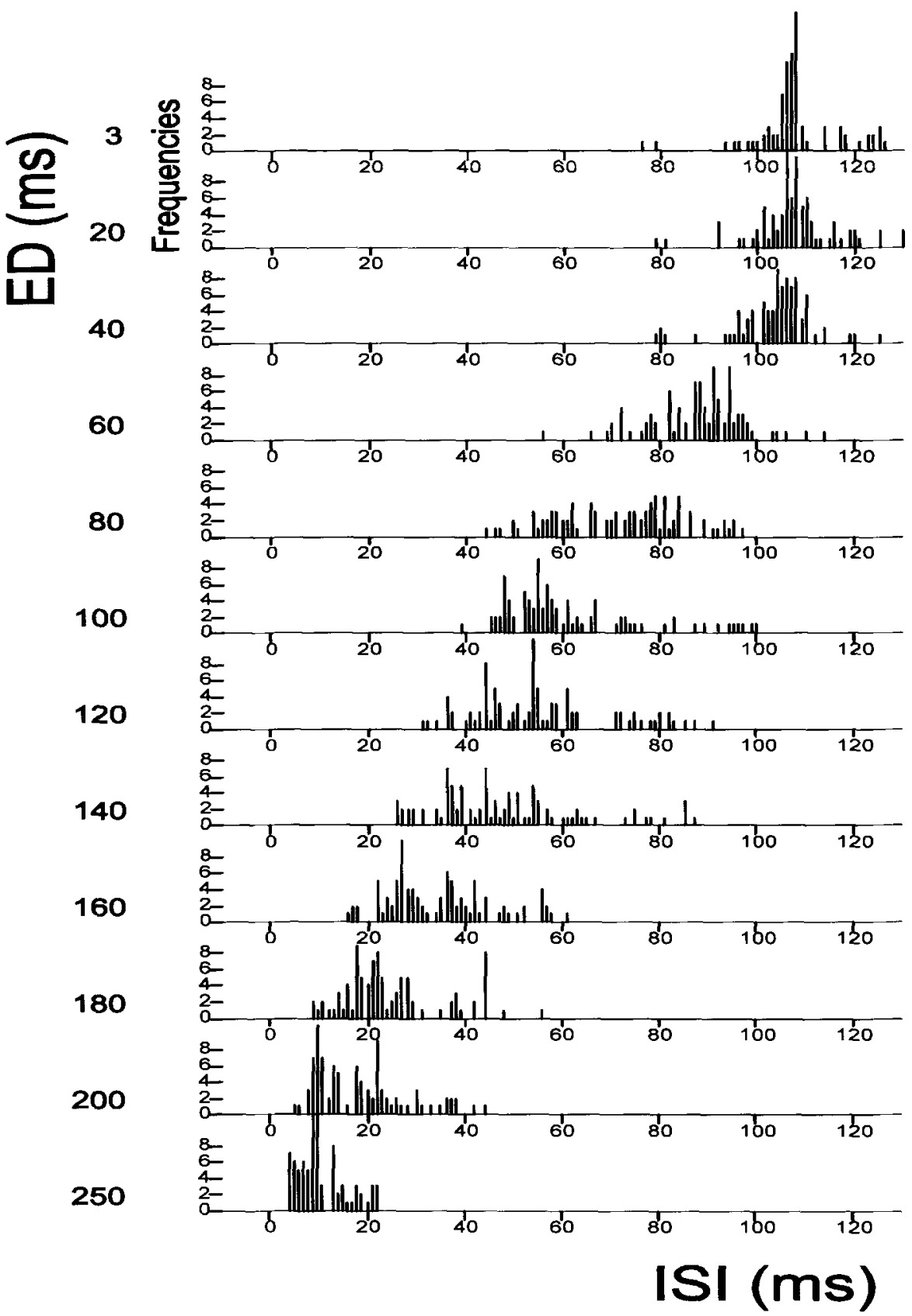

Figure 7C. Frequencies of critical interstimulus intervals (ISIs) for different exposure durations (EDs) and an angular separation of $12^{\circ}$, collapsed over subjects.

size in steps of $0.1 \mathrm{msec}$. In Figure $10, \chi^{2}$ is plotted as a function of period duration. A minimum is obtained at a period duration close to $4.5 \mathrm{msec}$. This value agrees surprisingly well with the hypothesized time quantum $T Q_{0}$ of $4.57 \mathrm{msec}$. The multimodal form of the distribution seems to support and specify the assumption that a quantal period of the asserted order of magnitude (with some stochastic fluctuations) plays the role of a minimum functional refractory period by which the breakdown of seen movement can be shifted in time. However, in contrast to the prominent modes of the original distribution, which signify strong commonalities among subjects, the de- scribed periodicity concerns only a small part of the total variance. The interpretation in terms of quantal processing has, therefore, to be viewed with caution and requires closer inspection of the entire pattern of results. A relevant aspect follows directly from the coherence limitation, as was illustrated in Figure 1, which results as a final breakdown from a gradual loss of synchrony among component oscillations. For increasing critical ISIs, this predicts increasing fuzziness of timing. To check on this property, three separate difference distributions were computed by splitting the raw data into subsets corresponding to subranges of ISIs of $<30 \mathrm{msec}, 30-69 \mathrm{msec}$, 


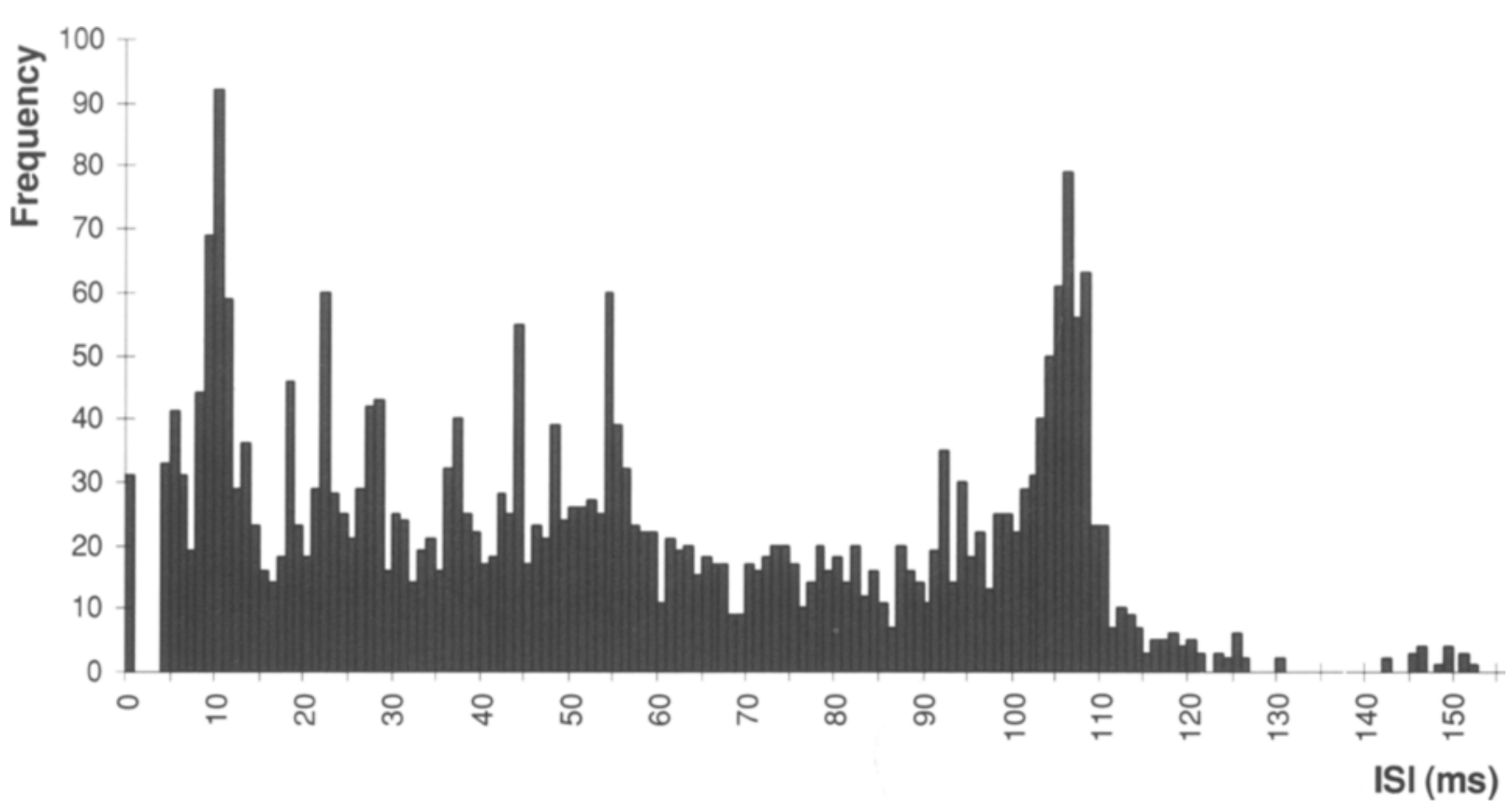

Figure 8. Frequencies of critical interstimulus intervals (ISIs) for simultaneity thresholds in beta movement for 46 subjects, collapsed over subjects, exposure durations, and angular separations.

and $>70 \mathrm{msec}$, respectively. The resulting histograms (Figure 11) show clear periodicities for the smaller ISI ranges and the expected wash-out for high ISIs. Yet, although the width of the distribution around the first peak in Figure 9 is small, as compared with standard deviations commonly reported for similar situations, it is at least twice as large as that expected from the TQM rationale assuming that random variations are of the order of $T Q_{0}$. Very. likely, this is caused by a mixture of random variations resulting from TQM-related fuzziness and technically induced random measurement errors, the latter being said to amount to about $2 \%-3 \%$ of the measured period. Unfortunately, separation of these two types of errors proves difficult, because both should increase in proportion to ISI. Clearly, however, the broadening of the distribution in the middle ISI range, as compared with the smallest range, should not be caused by measurement errors alone, inasmuch as it goes along with the appearance of new side maxima that agree with TQM predictions. Argument for the assumption that most of the overall variance is due to interindividual variation follows from the distribution of intraindividual $(S D s)$ given in Figure 12. In particular, extremely low $S D$ s of the order of 2-3 msec of 5 subjects testify that only a very limited part of variance can be due to technical reasons. A final resolution of this issue depends on technical improvements, left to future investigations.

\section{GENERAL DISCUSSION}

So far, we have considered beta motion merely as a paradigm suitable to check on temporal regularities predicted by TQM. We now discuss the results within the context of real-time aspects of sensory-cognitive processing. In section 1, we ask for possible conclusions about the temporal architecture of apparent motion itself. Section 2 concerns consequences for further possible developments of TQM as a general framework for neural realtime dynamics.

\section{Steps Toward Dynamic Interpretations}

If the regularities stated by TQM reflect properties rooted in the architecture of mental processes, they should offer tools for the interpretation of dynamic phenomena in real time and should prove to represent an appropriate point of departure for the construction of dynamic models. In this section, therefore, an attempt will be made to bridge the gap between quantal surface features and the specific dynamics of the phenomenon at issue. In general, real-time modeling on the basis of quantal assumptions will not be a one-step venture and will involve several levels of explanation. In the present context, we shall confine ourselves to one of these levels by considering quantal timing as given and asking, on this basis, for the most elementary prerequisites that must be assumed, to account for the pattern of empirical results.

From this perspective, the most important result of the experiment is certainly the finding that quantization holds for ISI rather than for SOA (= ED + ISI). This property provides an interesting example of how temporal quantization can offer arguments for a discussion of underlying processing structures.

As was mentioned above, adding EDs to ISIs completely destroys "reshuffling" regularities. This invalidates any straightforward formation-time hypothesis for the integration of stimuli into a motion percept (Kolers, 1972), 


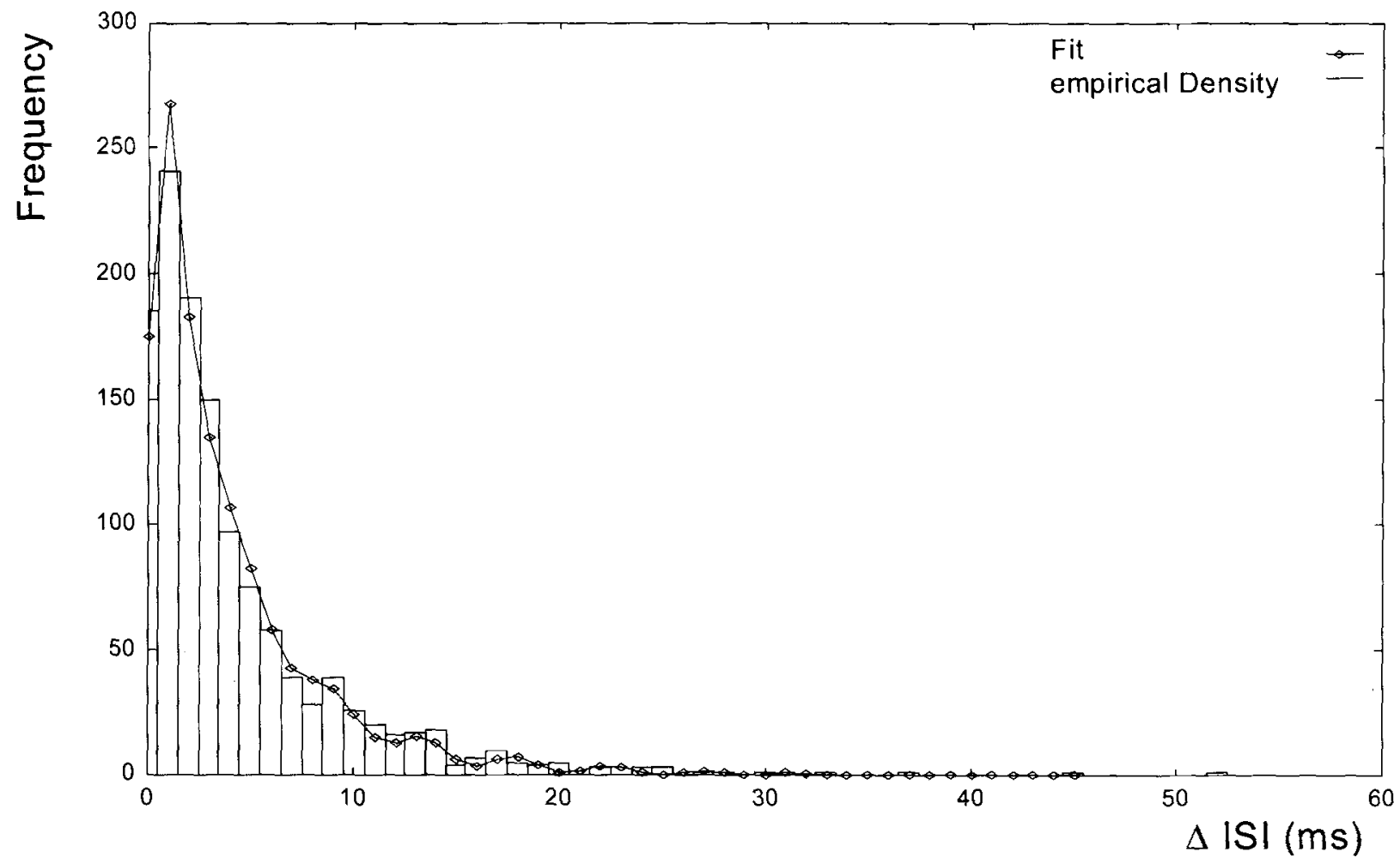

Figure 9. Distribution of differences of critical interstimulus intervals (ISIs) for pairs of simultaneity thresholds as measured under identical conditions of exposure duration and angular separation, collapsed over subjects and angular separations. Note that the column for zero differences corresponds to only one half of the interval of the original distribution. Circles indicate predictions from the fitted function.

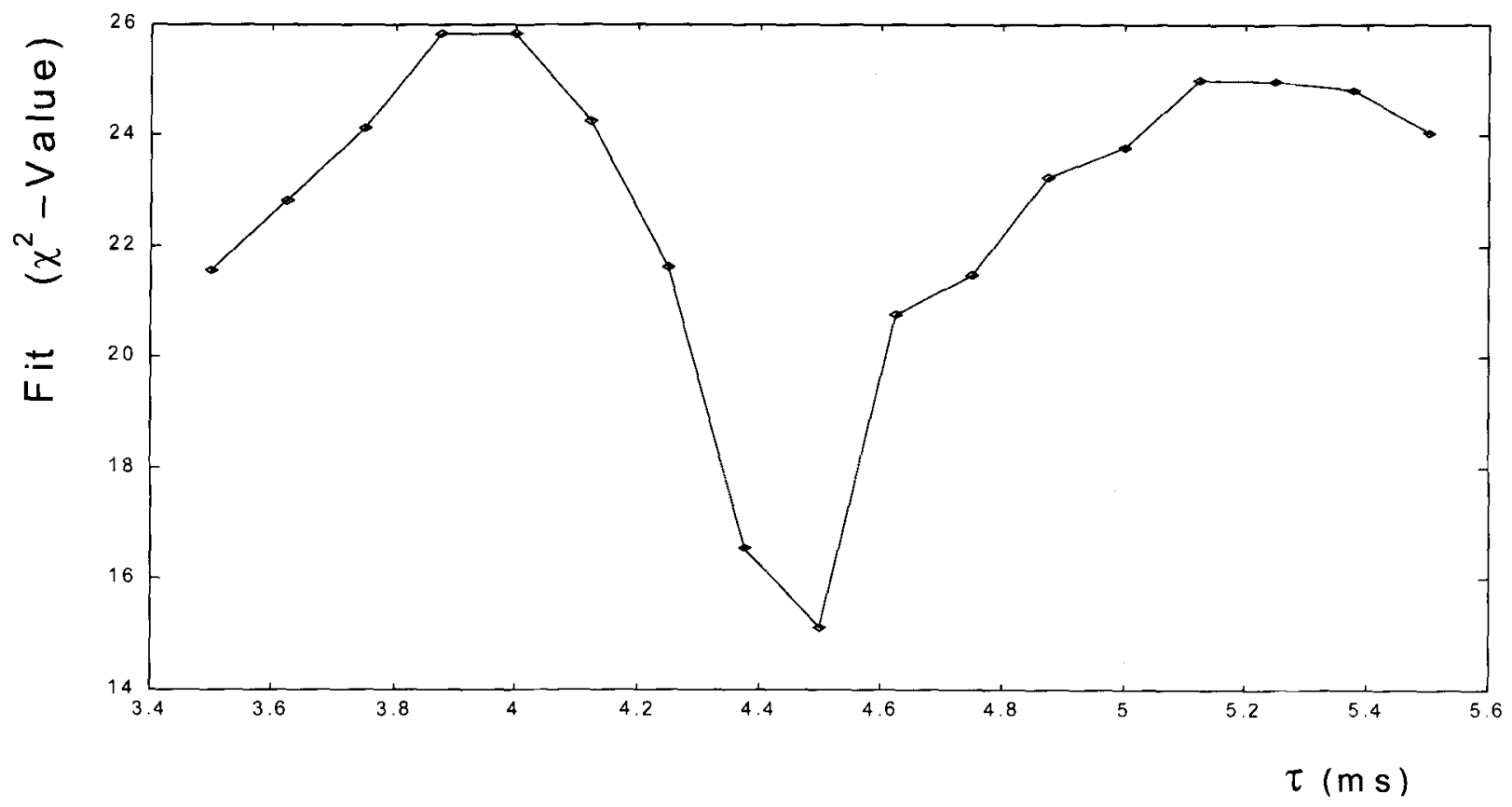

Figure 10. $\chi^{2}$ fits obtained for different period durations in the periodic part of the function used for fitting the overall difference distribution of simultaneity thresholds. $\tau$ denotes assumed period durations in milliseconds. 
A

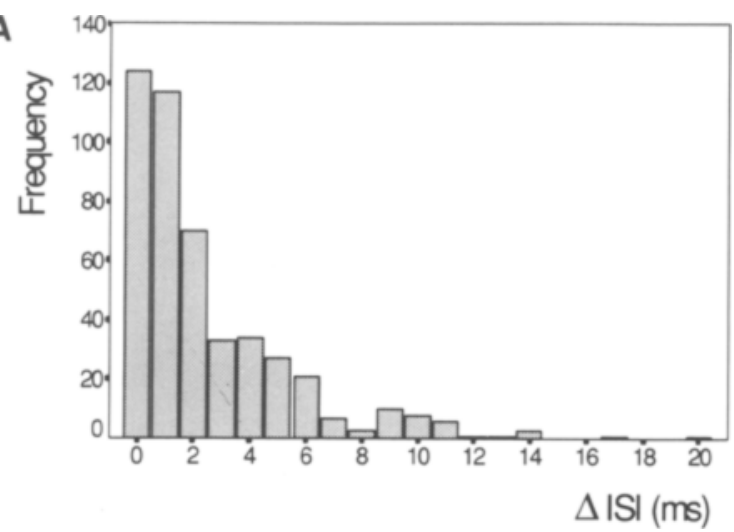

B

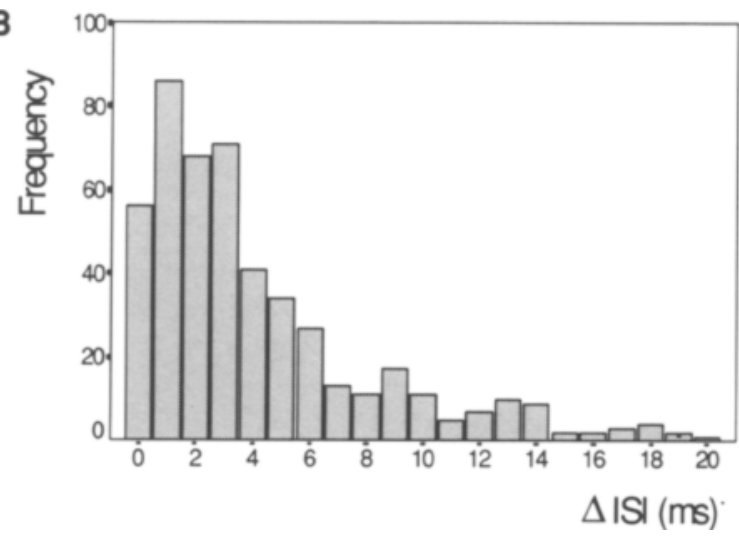

C

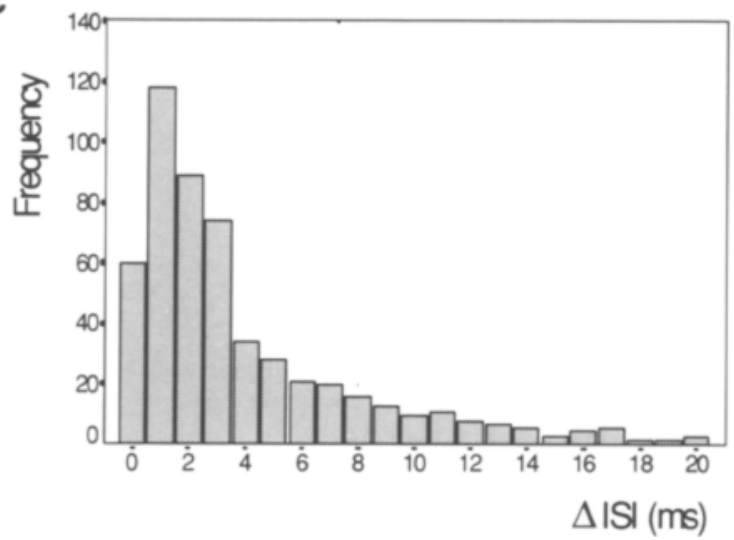

Figure 11. Difference distributions from the same data as Figure 9 (from top to bottom) for the lower (A), middle (B), and upper critical ranges $(C)$ corresponding to interstimulus intervals (ISIs) of $<30 \mathrm{msec}$, between 30 and $69 \mathrm{msec}$, and $>70 \mathrm{msec}$, respectively. Further explanations in the text.

at least for the conditions of continuous sequential alteration investigated. From a functionalist point of view, there is nothing surprising about the relevance of ISI, since in real movement, ISI corresponds to the time necessary for an object to move from one position to the other. To account for temporal quantization, one merely has to assume a quantal time threshold - that is, some quantal time period $t$ elapsed after offset of the first stimulus within a half-cycle, below which a second stimulus stops producing a motion percept. However, a serious difficulty for interpretations purely in terms of interpolated motion arises from the dependence of critical ISI on ED, because the time an object stays in a place has no plausible implication for its physical motion. Note that, qualitatively, the observed variation of critical ISI as a function of ED fulfills the classical expectation about visual persistence, which typically is found to decrease with increasing stimulus ED (Coltheart, 1980; see, also, Patterson, 1990).

In the vein of persistence conceptions, this property can be interpreted to mean that the start of integration is delayed until a static representation of the stimulus has decayed. Let $T^{*}$ denote the time measured from the onset of the first stimulus for which this is the case. Then, the assumption implies that threshold $T$ lies in the vicinity of the smallest quantal magnitude $T_{m}$ such that $T_{m}>T^{*}$. From a plain perceptual moment hypothesis of persistence (Patterson, 1990), one would therefore suppose $T^{*}$ to be constant as long as $T^{*}>\mathrm{ED}$, and otherwise to equal ED. Unfortunately, although the predictions of this flow model are qualitatively correct, they are not consistent with the quantitative structure of the data - namely, according to experimental evidence (cf. Figures 7A-7B), $T^{*}$ should, for the smallest EDs, roughly take a value between 55 and $105 \mathrm{msec} . T^{*} \sim 105 \mathrm{msec}$ thus turns out to be the most optimistic estimate. Therefore, at the latest for EDs within the range of $100-120 \mathrm{msec}, T^{*}$ should be located at the lower bound of the ISI. In other words, critical ISIs should reach their lower limit. In fact, however, they approach their lower limit only at EDs of about $180 \mathrm{msec}$, and concentration of occurrences at the lowest values slightly increases even beyond this point.

How are these results to be accounted for? At first glance, from the point of view of temporal quantization, it seems difficult to account for this complex relation without making unfounded assumptions. To find an appropriate construct, we have to keep in mind that formationtime accounts are certainly not entirely inadequate. The essence of these approaches consists in the assumption of processes involving the temporal growth and decay of strength parameters. These are, therefore, quite different in nature from processes that directly serve the timing of motion percepts and determine the fine-grained patterns of critical ISIs. Processes of the first type may reflect relatively slow processes of competition between motion and nonmotion interpretations of displays, which corresponds to rivalry and switching of activity between different neuron populations in areas of the visual pathway, whereas the latter probably reflect fast integration through binding within populations in the central areas of vision.

At this point, it is worth noting that, despite the indicated functional distinction, the introduction of components of continuous growth does not necessarily contradict the generality of quantal timing. In fact, as was demonstrated in simulations, autocatalytic growth and decay of strength parameters may be based on traces of strictly quantal lifetimes, without any trait of temporal quantization surfacing in the course of conscious perception (Alt- 


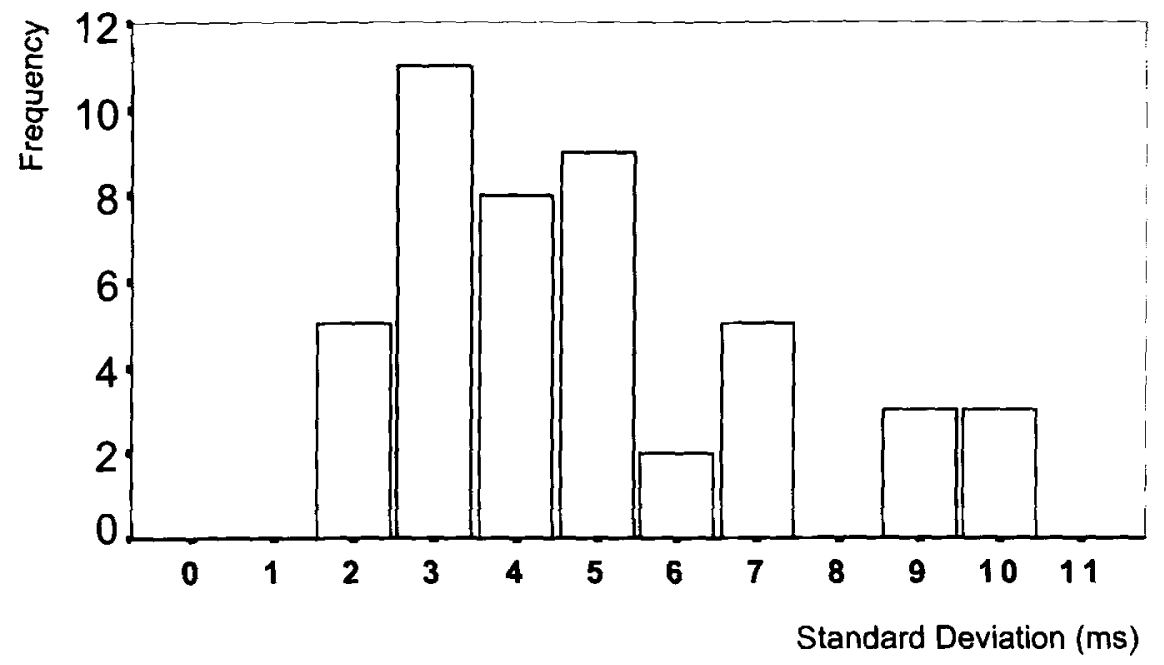

Figure 12. Distribution of standard deviations of the individual distributions of differences of critical interstimulus intervals (ISIs) between first trials and replications. Further explanations in the text.

mann, 1997). Generally, for dynamic real-time implementations of TQM, we expect a lack of explicit discreteness of outputs in the majority of input conditions.

Figure 13 illustrates the performance of a simple growth model based on similar considerations. Two quantal time barriers $\left(T_{1}, T_{2}\right.$, as an example) qualitatively account for the experimentally observed ISI trend. One basic characteristic is the differential growth and decay of a strength parameter of a nonmotion (static) interpretation of the first stimulus, S1. Time barriers are supposed to be entrained by the offset signal of S1. Broken lines in the upper part of Figure 13 were drawn to indicate possible quantal variations in actual termination times by small multiples of $T Q_{0}$. In the model, the differential effect of ED is caused by the nonmonotone trend of the assumed strength parameter. For a long exposure duration, $E_{2}$, and strength close to saturation at stimulus offset, there is an immediate decline of strength of the static interpretation of the stimulus, and consequently, integration with the second stimulus to form a motion percept is possible up to small ISIs represented by the time barrier $T_{2}$. For short exposure $E_{1}$, there follows first a period of further growth of strength after offset of S1 before it decreases and eventually drops below threshold. The ISI time window between upward and downward traversing of the threshold level is supposed to represent the zone of possible breakdowns of motion percepts, which, in the example, is assumed to occur at $T_{1}$.

The sketched scheme reproduces the reversal of order between EDs and ISIs and accounts for the fact that the second stimulus must not come outside a critical ISI interval, if a percept of motion is to result. In addition, the scheme correctly predicts that motion may be seen even for negative ISIs, if EDs are sufficiently long. Of course, there are still obvious weaknesses in the model in its present simple form. For example, the model does not explicitly account for the transition between two interpre- tations of stimulus displays and cannot explain why, at larger ISIs, the impression of motion may vanish again. Essentially, to incorporate such issues, it seems that a more elaborate version of the model will have to assume complementary stochastic accumulation processes for competing interpretations of stimulus displays. Also, the model, as it stands, is no more than a simplistic description, suitable only for the discussion of qualitative properties. There is no doubt that true modeling would have to explain in detail the linking between quantal oscillatory activity and timing in apparent movement. On the most general level, this requires a dynamic theory of synchronization, desynchronization, and rapid resynchronization of cortical activities predicting the quantal properties, of which TQM provides a semi-empirical account. An extremely interesting approach on which such an intention could build was recently advanced by Grossberg and Grunewald (1997), who described a network that quantitatively simulates perceptual framing data-in particular, data about temporal order judgments.

\section{The Time Quantum Model and the Temporal Architecture of Neural Mass Dynamics}

Although the reported findings may lead to an interesting modification of present theorizing on apparent motion, they appear to be by far more challenging to ideas about perceptual dynamics and its neural basis. Two major issues in this context are the fine-grained patterning of timing on the millisecond level and its assumed relatedness to superordinate range structures.

As to the patterning of timing, three findings seem to be of concern: the appearance of ultra-short periods of the order of a few milliseconds, the high agreement among individuals as to the duration of preferred periods, and the absolute level of ultra-precise timing. Although a quantal period of about $4.5 \mathrm{msec}$ is noticeably smaller than critical periods that are commonly accepted 


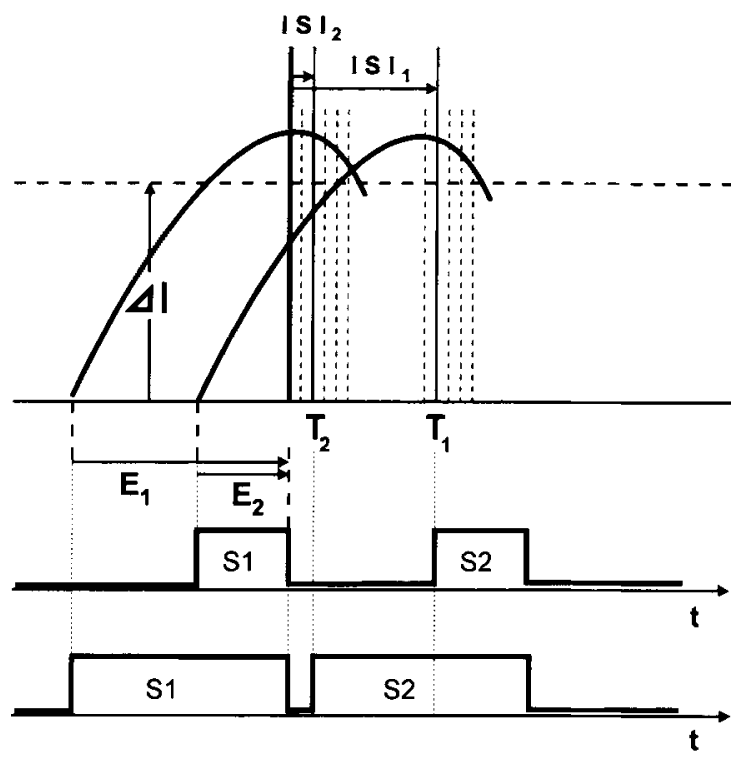

Figure 13. Schematic explanation of discrete critical interstimulus intervals (ISIs) occurring as a function of continuous variation of exposure duration (ED). Curves illustrate the growth and decay of strength values of nonmotion (static) interpretations of stimulus S1 for two exposure durations ED1 and ED2. The differential effect of ED on perceived movement is assumed to be caused by the nonmonotone trend of the strength parameter. For long exposure duration $E_{2}$ and strength close to saturation at stimulus offset, there is an immediate decline of strength of the static interpretation of the stimulus below the threshold $\Delta I$, and consequently, integration with the second stimulus to form a motion percept is still possible for small ISIs, in the example represented by the time barrier $T_{2}$. For short exposure $E_{1}$, there follows first a period of further growth of strength after offset of S1 before it decreases and eventually falls below threshold. The ISI time window between upward and downward traversing of the threshold level is supposed to represent the zone of possible breakdowns of motion percepts, which in the example in the special case, is assumed to occur at $T_{1}$. Note that critical ISIs reverse the order of the EDs. Broken lines indicate possible quantal variations in actual termination times.

as characteristics of human perception and cognition, which are of the order of some $20-200 \mathrm{msec}$, it is not below physiologically reasonable magnitudes. In fact, as reported above, delays caused by neuron refractivity and interneuronal transmission in the cortex are of the same order, and the duration of the time quantum $T Q_{0}$ compares well with occasionally determined peripheral (see, e.g., Fabiani, Schmer, Tait, Gafni, \& Kinarti, 1979) and central (Maltseva \& Masloboev, 1997) epochs of global electrical potentials. Fast oscillations with period durations compatible with the assumed quantum have recently been demonstrated to exist in the hippocampus (Buzsaki, Horváth, Urioste, \& Wise, 1992), an area known to be involved in the coordination of various cognitive activities within the cortex. What seems difficult to accept is the high intraindividual stability and, even more, the interindividual agreement of preferred periods at integer multiples of this duration, which is a consequence of the plain fact that prominent peaks appear in the group histogram that otherwise would have washed out. Interestingly, and from a psychological point of view particularly convincing about the results, this agreement is not at variance with strongly idiosyncratic courses of critical ISIs, considered as functions of ED as the independent variable. However, from a neurobiological point of view, this stability and the extreme precision of timing in sensorycognitive processing that we are forced to accept is felt to be the most striking outcome of the present investigation. According to the evidence put forward, precision must reach the 1-msec level, or even better it. Otherwise, neither sharp maxima for ISIs $>9 \mathrm{msec}$ in the original ISI distribution nor those in the difference distributions maxima at 0 ISI with $S D$ s below $3 \mathrm{msec}$ for some individuals could have occurred. For stability and precision of timing of global oscillatory activity in the cortex, this is an order of magnitude that does not easily fit in with commonly reported broad distributions in the beta and gamma bands (cf., however, Jürgens, Rösler, Hennighausen, \& Heil, 1995) and certainly represents a challenge to present-day biological theory and methods of measurement. Timing on such an ultra-precise level is bound to rely on synergetic mass effects of cooperating neuron populations (see Abeles, Prut, Bergman, \& Vaadia, 1994). Although a temporal precision of this level has not been demonstrated for visual pathways (see Maunsell \& Gibson, 1992, for measurements in primates), it does not seem surprising, given that mechanisms exhibiting a precision even beyond this level are well known from auditory spatial localization, where the largest between-ear time difference of sounds arriving from a source in lateral position and remaining fused as one subjective event is about $2 \mathrm{msec}$, whereas the smallest difference reported to give rise to a discriminable lateralization is about $10 \mu \mathrm{sec}$ (see, e.g., Howard, 1974). It remains a peculiarity of the case at issue that the observed precision in motion perception cannot be accomplished on the basis of relations between corresponding points on the time axis, as for phase shifts of periodic stimuli in the perception of spatial direction. Instead, the critical information must be signaled through the relation between off- and on-responses elicited by pairs of sequential stimuli. An extra difficulty with direct proof of this property could arise from the possibility that behaviorally successful processing may be accomplished by subpopulations of neurons that constitute merely a small fraction of the related activity that can be measured. It is to be hoped that further behavioral analysis will provide guidance for progress in substraterelated analysis.

The second issue is that of ranges suggested to span adaptive frames of reference for timing. Although neural equivalents of ranges of preferred periods have rarely ever been considered explicitly, TQM provides some hints as to how this could be accomplished. For this, we have to realize that, as an automatic consequence of the hierarchy formation constraint, ranges fall apart into subranges, with the defining property that harmonic relations do exist only between and not within subranges (see, also, Geissler, 
1997). For $M=30$, confining to $1: 2$ ratios between period durations, there are exactly four such subranges. The first of these subranges extends from multiples $k=16$ to $k=30$ of $T Q_{0}$; the second, third, and fourth extend from $k=15$ to $k=8, k=7$ to $k=4$, and $k=3$ to $k=2$, respectively. As a common factor, $k=1$ is unique. In this sequence of subranges, the elements of the first subrange always constitute the top periods of possible hierarchies, which become segmented by subordinate periods. Within those, $k=30$ represents an absolute upper bound, and the remaining elements represent possible operative bounds (see the introduction). In general, one can say that the first subrange completely determines the possible hierarchies that can be established. Adopting $T Q_{0}=4.57 \mathrm{msec}$, this subrange corresponds to the interval $73 \leq t \leq 137$ of period durations $t$ in milliseconds.

Crucial for application to neural correlates seems the surprising fact that it is this very interval that corresponds to the alpha band of brain activity. In fact, although suggested on a purely behavioral basis, after conversion into frequencies, it yields the interval $7.3 \leq f \leq$ 13.7 in hertz, which coincides with the conventional definition of the alpha band. In pointing this out in Geissler (1991, 1997), it was, of course, not claimed that "idling" spontaneous alpha oscillations represent a direct equivalent of information processing. However, the prominent position of $R_{1}$ and experimental findings about relations between individual characteristics of the alpha band and of short-term memory performance led us to suggest that oscillations from this range of frequency might play an important, although indirect, role in cognition, controlling faster oscillations through resonance. Preliminary results of psychophysiological investigations seem to support this conclusion (Geissler, 1997).

The above issues of timing also appear to have a bearing on the further development of TQM as a global framework for describing the real-time dynamics of natural neural networks. For relating TQM constructs to common notions of brain oscillations, it seems of utmost importance to note that they refer to the time domain and will not completely translate into a language of frequency, as is central to most contemporary psychophysiological approaches and techniques. This holds true of period as the most basic concept of TQM, which does not imply the existence of a unique correspondence to a period of a sinusoidal oscillation. Things are even more complex for the range concept, as coherence limitations imply nonstationary processes and a single period does not have meaning in terms of one single frequency. For a reconciliation with physiological notions, we rather have to concentrate on properties of stable oscillatory events, such as zero crossings, that are not altered by a certain degree of chaotic perturbation that, in the frequency domain, would add power in a broad range of the spectrum. Potentially interesting specimens are stable oscillations within spindle-like structures, as were demonstrated by Williamson, Kaufman, Lu, Wang, and Karron (1997) in spontaneous alpha activity, and chaotic high- frequency oscillations from local field derivations in the hippocampus, demonstrated by Buzsaki et al. (1992). The latter yielded two bands, one somewhat above 200 zero crossings per second, the other above 100 -that is, period durations of the order of $T Q_{0}$ and $2 T Q_{0}$. The fine structure of these oscillations showed "exact" halving segmentations, in the sense that always one additional zero crossing of the faster oscillation falls into the periods between two zero crossings of the slower. This result points to cyclic organization within and between layers of neurons as a possible mechanism of hierarchy formation among periods.

\section{REFERENCES}

Abeles, M., Prut, Y., Bergman, H., \& VaAdia, E. (1994). Synchronization in neuronal transmission and its importance for information processing. In G. Buzsaki, R. Llinás, W. Singer, A. Berthoz, \& Y. Christen (Eds.), Temporal Coding in the Brain, 39-50. Berlin: Springer-Verlag.

Altmann, B. (1997). Zur Simulation dynamischer Phänomene der visuellen Wahrnehmung [Simulation of dynamic phenomena in visual perception]. Unpublished doctoral dissertation, University of Leipzig.

Anstis, S. M. (1978). Apparent movement. In R. Held, H. W. Leibowitz, \& H.-L. Teuber (Eds.), Handbook of sensory physiology (Vol. 8, pp. 655-673). Berlin: Springer-Verlag.

Anstis, S. M. (1980). The perception of apparent movement. Philosophical Transactions of the Royal Society of London: Series B, 290, 153-168.

ANSTIS, S. M. (1986). Motion perception in the frontal plane: Sensory aspects. In K. R. Boff, L. Kaufmann, \& J. P. Thomas (Eds.), Handbook of perception and human performance: Vol. I. Sensory processes and perception (chap. 16, pp. 1-27). New York: Wiley.

Anstis, S. M., Giaschi, D., \& Cogan, A. I. (1985). Adaptation to apparent motion. Vision Research, 25, 1051-1062.

BAER, K.V. von (1864). Welche Auffassung der lebendigen Natur ist die richtige? Und wie ist diese Auffassung auf die Entomologie anzuwenden? [Which view of living nature is correct? And, how is this view to be applied to entomology?]. In H. Schmitzdorf (Ed.), Reden, gehalten in wissenschaftlichen Versammlungen, und kleine Aufsätze vermischten Inhalts (pp. 237-283). St. Petersburg: Verlag der Kaiserlichen Hofbuchhandlung.

BÉkÉsy, G. VON (1936). Über die Hörschwelle und Fühlgrenze langsamer sinusförmiger Luftdruckschwankungen [On thresholds for hearing and feeling of sinusoidal low-frequency air pressure oscillations]. Annalen der Physik, 26, 554-566.

BLock, R. A. (1990). Model of psychological time. In R. A. Block (Ed.), Cognitive models of psychological time (pp. 1-58). Hillsdale, NJ: Erlbaum.

BRADDICK, O. (1974). A short-range process in apparent motion. Vision Research, 14, 519-527.

BRADDICK, O. (1980). Low-level and high-level processes in apparent motion. Philosophical Transactions of the Royal Society of London: Series $B, 290,137-151$.

BREDENKAMP, J. (1993). Die Verknüpfung verschiedener Invarianzhypothesen im Bereich der Gedächtnispsychologie. [The connection of various invariance hypotheses in the psychology of memory]. Zeitschrift für Experimentelle und Angewandte Psychologie, 11, 368-385.

BRYSBAERT, M. (1994). Behavioral estimates of interhemispheric transmission time and the signal detection method: A reappraisal. Perception \& Psychophysics, 56, 479-490.

Buzsaki, G., Horváth, Z., Urioste, R., \& Wise, K. (1992). Highfrequency network oscillations in the hippocampus. Science, 256, 1025.

Coltheart, M. (1980). Iconic memory and visible persistence. Perception \& Psychophysics, 27, 183-228.

DeHAENe, S. (1993). Temporal oscillations in human perception. Psychological Science, 4, 264-269. 
EISLER, H. (1990). Breaks in the psychophysical function for durations. In H.-G. Geissler, M. H. Müller, \& W. Prinz (Eds.), Psychophysical explorations of mental structures (pp. 242-252). Toronto: Hogrefe \& Huber.

EXNER, S. (1875). Experimentelle Untersuchungen der einfachsten Psychischen Prozesse [Experimental investigations into the simplest mental processes]. Pflügers Archiv für die gesamte Physiologie, 11, 403-432.

Fabiani, M., Schmer, H., Tait, C., Gafni, M., \& Kinarti, R. (1979). A functional measure of brain activity: Brain stem transmission time. Electroencephalography \& Clinical Neurophysiology, 47, 483-491.

FINLAY, D., \& VON GRÜNAU, M. (1987). Some experiments on the breakdown effect in apparent motion. Perception \& Psychophysics, 42, 526-534.

FRAISSE, P. (1966). Zeitwahrnehmung und Zeitschätzung [Perception and judgment of time]. In W. Metzger (Ed.), Handbuch der Psychologie (Vol. 1, pp. 656-690). Göttingen: Hogrefe.

FreEMAN, W. J. (1964). A linear distributed feedback model for prepyriform cortex. Experimental Neurology, 10, 525-547.

FreEMAN, W. J. (1972). Measurement of open-loop responses to electrical stimulation in olfactory bulb of cat. Journal of Neurophysiology, 35, 745-761.

FrEeman, W. J. (1974). Stability characteristics of positive feedback in aneural population. IEEE Transactions on Biomedical Engineering, 21, 358-364.

GANZ, L. (1975). Temporal factors in visual perception. In E. C. Carterette \& M. P. Friedman (Eds.), Handbook of perception: Vol. V: Seeing (pp. 169-232). New York: Academic Press.

GEISSLER, H.-G. (1985a). Sources of seeming redundancy in temporally quantized information processing. In G. d'Ydewalle (Ed.), Cognitive information processing and motivation (Vol. 3, pp. 199-228). Amsterdam: North-Holland.

GEISSLER, H.-G. (1985b). Zeitquantenhypothese zur Struktur ultraschneller Gedächtnisprozesse [Time quantum hypothesis on the structure of ultra-speed memory processes]. Zeitschrift für Psychologie, 193, 347-362.

GeissLER, H.-G. (1987). The temporal architecture of central information processing: Evidence for a tentative time-quantum model. Psychological Research, 49, 99-106.

GeIssLer, H.-G. (1990). Foundations of quantized processing. In H.-G. Geissler, M. H. Müller, \& W. Prinz (Eds.), Psychophysical explorations of mental structures (pp. 193-210). Toronto: Hogrefe \& Huber.

GEISSLER, H.-G. (1991). Zeitcodekonstanten-ein Bindeglied zwischen Psychologie und Physiologie bei der Erforschung kognitiver Prozesse? Hypothesen und Überlegungen zu Quantenstrukturen in der AlphaAktivität des Gehirns.[Time code constants-a link between psychology and physiology in the investigation of cognitive processes? Hypotheses and deliberations on quantal structures in the alpha activity of the brain]. Zeitschrift für Psychologie, 199, 121-143.

GeIssLER, H.-G. (1992). New magic numbers in mental activity? On a taxonomic system for critical time periods. In H.-G. Geissler, S. W. Link, \& J. T. Townsend (Eds.), Cognition, information processing and psychophysics (pp. 293-322). Hillsdale, NJ: Erlbaum.

GEISSLER, H.-G. (1997). Is there a way from behavior to nonlinear brain dynamics? On quantal periods in cognition and the place of alpha in brain resonances. International Journal of Psychophysiology, 26, 381-393.

GrossBerG, S., \& Grunewald, A. (1997). Cortical synchronization and perceptual framing. Journal of Cognitive Neuroscience, 9, 117-132.

HENDRICKSON, A. E. (1972). An integrated molar/molecular model of the brain. Psychological Reports, 30, 343-368.

HIRSCH, I. J. (1975). Temporal aspects of hearing. In B. Tower (Ed.), The nervous systems: Vol. 3. Human communication and its disorders. New York: Raven.

HirSCH, I. J., \& SHERrick, C. E. (1961). Perceived order in different sense modalities. Journal of Experimental Psychology, 62, 423-432.

Howard, I. P. (1974). The spatial senses. In E. C. Carterette \& M. P. Friedman (Eds.), Handbook of perception: Vol. III. Biology of perceptual systems (pp. 273-290). New York: Academic Press.
JoKEIT, H. (1990). Analysis of periodicities in human reaction times. Naturwissenschaften, 77, 289-291.

Jürgens, E., Rösler, F., Hennighausen, E., \& Heil, M. (1995). Stimulus-induced gamma oscillations: Harmonics of alpha activity? NeuroReport, 6, 813-816.

Kandel, E. R., Schwartz, J. H., \& Jessell, T. M. (1995). Principles of neural science (3rd ed.). London: Prentice-Hall.

KolERS, P. A. (1964). The illusion of movement. American Sciences, 211 , 98-106.

Kolers, P. A. (1972). Aspects of motion perception. Oxford: Pergamon Press.

KrISTOFFERSON, A. B. (1980). A quantal step function in duration discrimination. Perception \& Psychophysics, 27, 300-306.

Kristofferson, A. B. (1990). Timing mechanisms and the threshold for duration. In H.-G. Geissler (Ed.) in collaboration with M. H. Müller and W. Prinz, Psychophysical explorations of mental structures (pp. 269-277). Toronto: Hogrefe \& Huber.

LaRsen, A., Farell, J. E., \& Bundesen, C. (1983). Short-range and long-range processes in visual apparent movement. Psychological Research, 45, 11-48.

LATOUR, P. L. (1967). Evidence of internal clocks in the human operator. Acta Pschologica, 27, 341-348.

Lisman, J. E., \& IDIART, M. A. P. (1995). Storage of $7 \pm 2$ short-term memories in oscillatory subcycles. Science, 267, 1512-1515.

MaltSEVA, I. V., \& MasLoboev, Y. P. (1997). Alpha-rhythm parameters and short-term memory span. International Journal of Psychophysiology, 26, 369-380.

MAUNSELl, J., \& GiBSON, R. J. (1992). Visual response latencies in striate cortex of the macaque monkey. Journal of Neurophysiology, 68, 1332-1343.

MCREYNOLDS, P. (1953). Thinking conceptualized in terms of interacting moments. Psychological Review, 60, 319-330.

NEFF, W. S. (1936). A critical investigation of the visual apprehension of movement. American Journal of Psychology, 48, 1-42.

Neuhaus, W. (1930). Experimentelle Untersuchungen der Scheinbewegung [Experimental investigations into apparent motion]. Archiv für die gesamte Psychologie, 75, 11-18.

NeumanN, O. (1983). Moment. In J. Ritter \& K. Gründer (Eds.), Historisches Wörterbuch der Philosophie (Vol. VI, pp. 108-114). Basel: Schwabe.

Patterson, R. (1990). Perceptual moment models revisited. In R. A. Block (Ed.), Cognitive models of psychological time (pp. 85-100). Hillsdale, NJ: Erlbaum.

PöPPEL, E. (1970). Excitability cycles in central intermittency. Psychlogische Forschung, 34, 1-9.

Ramachandran, V.S., \& Gregory, R. L. (1978). Does colour provide an input to human motion perception? Nature, 275, 55-56.

SARRIS, V. (1989). Max Wertheimer on seen motion: Theory and evidence. Psychological Research, 51, 58-68.

Shipley, W. C., KenNeY, F. A., \& KING, M. E. (1945). Beta apparent movement under binocular and interocular stimulation. American Journal of Psychology, 58, 545-549.

Stroud, J. M. (1956). The fine structure of psychological time. In $\mathrm{H}$. Quastler (Ed.), Information theory in psychology (pp. 140-207). Glencoe, IL: The Free Press.

Treisman, M., Cook, N., Naish, P. L. N., \& MacCrone, J. K. (1994). The internal clock: Electroencephalographic evidence for oscillatory processes underlying time perception. The Quarterly Journal of Experimental Psychology, 47A (2), 241-289.

vaN LeEUWEN, C., \& BAKKER, L. (1995). Stroop can occur without Garner interference: Strategic and mandatory influences in multidimensional stimuli. Perception \& Psychophysics, 57, 379-392.

WEISs, V. (1992). The relationship between short-term memory capacity and EEG power spectral density. Biological Cybernetics, $\mathbf{6 8}, 165$ 175.

WERTHEIMER, M. (1912). Experimentelle Studien über das Sehen von Bewegung [Experimental studies in seen motion]. Zeitschrift für Psychologie, 61, 161-256.

Williamson, S. W., Kaufman, L., Lu, Z.-L., Wang, J.-Z., \& Karron, D. 
(1997). Study of the human occipital alpha rhythm: The alphon hypothesis and alpha suppression. International Journal of Psychophysiology, 26, 63-76.

\section{APPENDIX}

\section{Test on Preferred Critical ISIs}

Statistical tests on empirically preferred ISIs, represented by peaks in the histogram of Figure 8, were based on a comparison with a uniform ISI distribution within the range $(0,120)$, in milliseconds. A histogram of $n$ independent trials conforming to this distribution will always exhibit peaks, because the probability of a flat histogram is close to zero. Although the density function of the frequency of events in a fixed bin is binomial, the maximum of the frequencies of all bins has a much more complicated structure. However, from the histogram, it is obvious that actually appearing peaks are wider than one bin. Therefore, the test performed was based on the maximum $k$ of the sums of any three subsequent bins $(k>3 n / 120)$. From simulations with 100,000 distributions of $n=3,000$ trials, the empirical CDF of $k$ was computed. The critical values of $k$ that were found are

$$
\begin{aligned}
& 106, p(k \geq 106)=.037<.05 \\
& 110, p(k \geq 110)=.0075<.01 \\
& 112, p(k \geq 112)=.003<.005 .
\end{aligned}
$$

We can, therefore, show that the following peaks are not by chance:

$$
\begin{array}{ll}
8,9,10, & l=228, p \ll .005 \\
106,107,108, & l=197, p \ll .005 \\
54,55,56, & l=131, p \ll .005 \\
21,22,23, & l=117, p<.005 \\
26,27,28, & l=113, p<.005 \\
42,43,44, & l=108, p<.05 \\
(4,5,6, & l=105, .05<p<.06) .
\end{array}
$$

The sum for the three subsequent bins is denoted by $l$. Note that the presence of 5 (6) such peaks in the same histogram is even more unlikely under the uniformity assumption.

\section{Fitting ISI Difference Distributions}

A mix of normal distributions was fitted to the overall distribution of ISI differences. Since the $S D$ s of the individual distributions are approximately gamma-distributed (see Figure 12), the density function of the resulting distribution was chosen to be

$$
g(x)=\frac{1}{\sqrt{2 \pi}} \int_{0}^{\infty} \frac{1}{\sigma} e^{\left(-\frac{x^{2}}{2 \sigma^{2}}\right)} \frac{b^{p}}{\Gamma(p)} \sigma^{p-1} e^{-b \sigma} d \sigma,
$$

where $p$ and $b$ had to be fitted. For 15 degrees of freedom the obtained $\chi^{2}$ value was 22.1 , with a corresponding $z$ value between .85 and .9 .

To include the periodic variations visible in the histogram, a periodic function $h(x)$ of period $\tau$ and an amplitude modulation $w(x)$ of a shape similar to a normal distribution was introduced:

$$
\begin{gathered}
h(x)=w(x) \cos \frac{2 \pi x}{\tau} \\
w(x)=\frac{1}{\frac{1}{N_{0 . \sigma}(x)}+\frac{1}{g(x)}} .
\end{gathered}
$$

The density function is the normed average of $g(x)$ and $h(x)$. The weight $w(x)$ has to be smaller than $g(x)$ to ensure that the resulting density function is nonnegative, this being the purpose of the formula on the right. The resulting fit is shown in Figure 11 in the text. The $\chi^{2}$ value could, in this way, be further reduced to 15.1 , which corresponds to a $z$ value of .5 to .6 .

(Manuscript received October 31, 1997; revision accepted for publication April 17, 1998.) 